\title{
THE LASER INTERFEROMETER GRAVITATIONAL WAVE OBSERVATORY PROJECT LIGO
}

\author{
JAMES KENT BLACKBURN \\ California Institute of Technology \\ Physics, Mathematics and Astronomy Division \\ LIGO Project, Mail Code 51-33 \\ Pasadena, CA 91125, U.S.A. \\ E-mail:kent@ligo.caltech.edu
}

\begin{abstract}
The Laser Interferometer Gravitational Wave Observatory (LIGO) will search for direct evidence of gravitational waves emitted by astrophysical sources in accord with Einstein's General Theory of Relativity. State of the art laser interferometers located in Hanford, Washington and Livingston Parish, Louisiana will unambiguously measure the infinitesimal displacements of isolated test masses which convey the signature of these gravitational waves. The initial implementation of LIGO will consist of three interferometers operating in coincidence to remove spurious terrestrial sources of noise. Construction of the facilities has begun at both sites, while research continues to design and develop the technologies to be utilized in achieving the target sensitivity curve having a minimum sensitivity of $\sim 1 \times 10^{-19}$ meters $/ \sqrt{\mathrm{Hz}}$ at $\sim 150 \mathrm{~Hz}$ for the initial phase of LIGO. Advanced LIGO interferometers of the future, having strain sensitivities on the order of $10^{-24} / \sqrt{\mathrm{Hz}}$ corresponding to optical phase sensitivities on the order of $10^{-11}$ radians $/ \sqrt{\mathrm{Hz}}$ over an observing band from $10 \mathrm{~Hz}$ to $10 \mathrm{kHz}$, require a complete understanding of the noise sources limiting detection. These fundamental noise sources will be quantitatively highlighted along with the principles of operation of the initial LIGO detector system and the characteristics of the most promising sources.
\end{abstract}

1. Introduction. The Laser Interferometer Gravitational Wave Observatory (LIGO) [1] is currently under joint development by the California Institute of Technology and the Massachusetts Institute of Technology and is funded by the National Science Foundation. The scientific aim of LIGO is the detection and study of cosmic gravitational waves. Sources of such waves include coalescing compact binary systems made up of neutron stars and black holes, supernovae, pulsars and the stochastic background of gravitational waves (the gravitational analog to the cosmic microwave background). Beyond these

1991 Mathematics Subject Classification: 83C35, 83B05.

The paper is in final form and no version of it will be published elsewhere. 


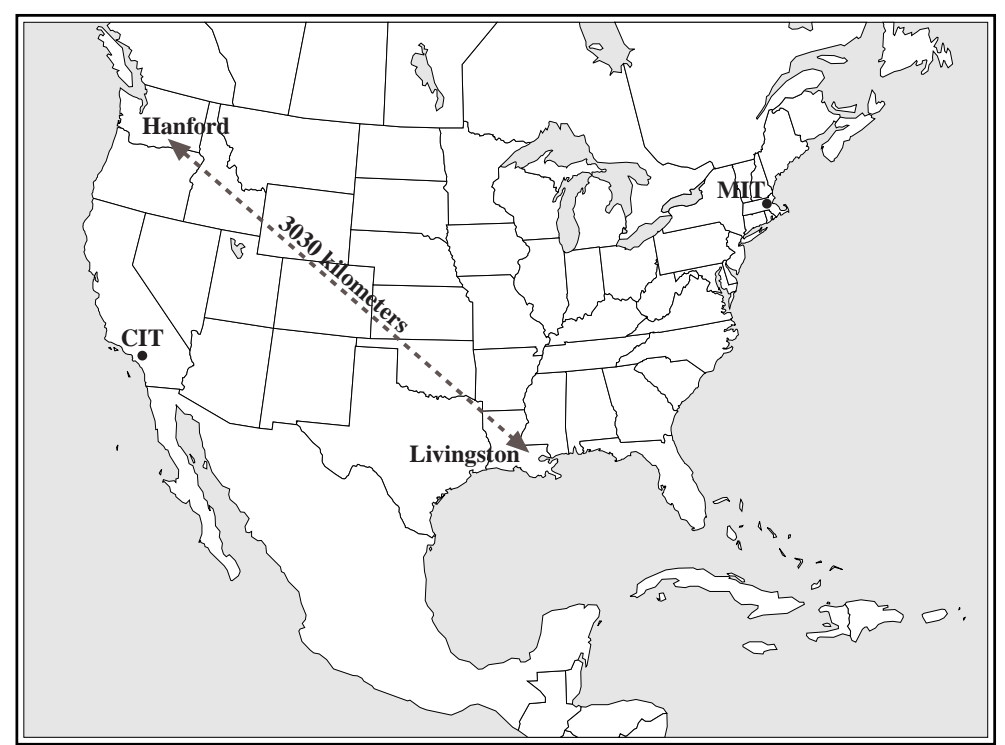

Figure 1: LIGO Sites: Two remote facilities will be located in Hanford, Washington and Livingston Parish, Louisiana. The distance between the sites is 3030 kilometers, corresponding to a maximum difference in the time of arrival of \pm 10 milliseconds for a gravitational wave.

known sources of gravitational waves awaits many great surprises as this new class of instrument opens a never before observed window on the universe.

Initially, LIGO will consist of three laser interferometers operating in coincidence. Two of these interferometers will be located at the Hanford, Washington site on the Department of Energy Hanford Nuclear Facility and the third will be located in Livingston Parish, Louisiana. The Hanford site will house within a common vacuum envelope, a full length 4 kilometer interferometer and a half length 2 kilometer interferometer. This ratio of $2: 1$ will aid in the rejection of non-gravitational signals by demanding the same ratio of the strain signal observed from a true signal. The Livingston site will house a single full length 4 kilometer interferometer providing the needed coincidence for unambiguous detection.

The vertex of the Hanford instrument is located at the geographic coordinates $46^{\circ} 27^{\prime} 18.5^{\prime \prime} N, 119^{\circ} 24^{\prime} 27.1^{\prime \prime} W$, with the arms oriented toward the northwest at a bearing of $N 36.8^{\circ} \mathrm{W}$ and the southwest at a bearing $S 53.2^{\circ} \mathrm{W}$. The vertex of the Livingston instrument is located at the geographic coordinates $30^{\circ} 33^{\prime} 46.0^{\prime \prime} N, 90^{\circ} 46^{\prime} 27.3^{\prime \prime} \mathrm{W}$, with the arms oriented toward the southeast at a bearing of $S 18^{\circ} \mathrm{E}$ and the southwest at a bearing $S 72^{\circ} \mathrm{W}$. The separation of the sites is 3030 kilometers, corresponding to a maximum difference in the time of arrival for gravitational waves at the two sites of \pm 10 milliseconds (see Figure 1). The arms of the interferometers between the two sites are oriented such that one arm of each interferometer makes the same angle relative to the great circle that passes through the vertices of the two sites. The second arm at each site is perpendicular to the first and lies very close to the local horizontal plane. This orientation provides for nearly maximum coincidence sensitivity to a particular gravitational wave polarization. 
Accurate and precise absolute timing resolution will be achieved by standardizing to the Global Positioning System (GPS) at the two sites. This will further allow the correlation of LIGO data with other types of detectors, such as resonant bar detectors, high energy particle detectors and electromagnetic astronomical observations. The separation between the two sites is sufficient to eliminate coincidental terrestrial perturbations. A gravitational wave signal will be correlated at the two sites, thereby verifying detection. In addition, both sites will use an environmental monitoring system to measure local terrestrial perturbations. This will improve the rejection of accidental coincidences and provide important diagnostic capabilities at the sites. When used in conjunction with the correlation between the two signals from the full length and half length interferometers at the Hanford site, the environmental monitoring system will set limits on the broad band search for stochastic background gravitational waves at the lowest frequencies observable by LIGO.

\begin{tabular}{|c|c|c|c|c|c|}
\hline Project & Country & $\mathbf{N}$ & Len $(\mathbf{k m})$ & Lat & Long \\
\hline \hline LIGO & U.S.A. (WA) & 2 & $4.0 \& 2.0$ & $46.45^{\circ} \mathrm{N}$ & $119.41^{\circ} \mathrm{W}$ \\
\hline LIGO & U.S.A. (LA) & 1 & 4.0 & $30.56^{\circ} \mathrm{N}$ & $90.77^{\circ} \mathrm{W}$ \\
\hline VIRGO & Italy/France & 1 & 3.0 & $43.53^{\circ} \mathrm{N}$ & $10.5^{\circ} \mathrm{E}$ \\
\hline GEO600 & Germany/Britain & 1 & 0.6 & $52.25^{\circ} \mathrm{N}$ & $9.81^{\circ} \mathrm{E}$ \\
\hline TAMA & Japan & 1 & 0.3 & $35.68^{\circ} \mathrm{N}$ & $139.54^{\circ} \mathrm{E}$ \\
\hline AIGO & Australia & 1 & 1.0 & $? ?$ & $? ?$ \\
\hline
\end{tabular}

Having two sufficiently separated sites, LIGO will be capable of making confident detections of gravitational waves. To fully study the scientific content of gravitational waves, LIGO is also planning to operate as a component in an international network of broad-band interferometric gravitational wave detectors. Long baseline interferometers for the detection of gravitational waves are expected to be in operation at the same time as LIGO by the VIRGO Project at Pisa, Italy and by the GEO600 Project at Hannover, Germany. Both Japan and Australia are making plans to establish long baseline interferometers. A global network of detectors, listed in the table above, will be able to fully study the wealth of information from gravitational waves, including details not possible by LIGO alone, such as the polarization and source position on the sky. Simultaneous detection by several global interferometers will improve the confidence and improve the overall signal strength. Resonant bar detectors are also expected to be on the global network located in Frascati, Italy; Baton Rouge, Louisiana; and Perth Australia, by the time of the inception of these interferometric detectors.

2. Gravitational waves. According to the theory of general relativity, compact massive objects such as neutron stars and black holes warp the geometry of space-time. When these objects experience an acceleration as is the case in a supernova or the inspiral of a compact binary system, the geometry of space-time experiences a dynamic change which propagates at the speed of light in the form of a gravitational wave [2]. Gravitational waves have yet to be detected directly, though their indirect influence has been observed 


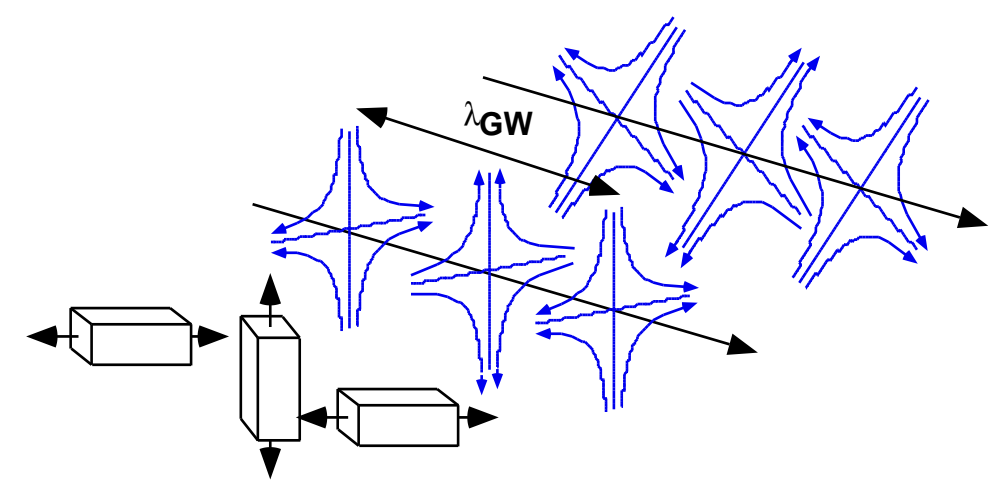

Figure 2: The distortion on a body that results from passage of a gravitational wave. The $h_{\times}$ polarization is shown on top and $h_{+}$polarizations is shown in the middle for this particular choice of coordinates.

with great accuracy in the binary pulsar PSR $1913+16$ by Russel Hulse and Joseph Taylor [3].

Exploring the universe through gravitational waves will reveal exciting new astrophysics not observable with electromagnetic radiation. LIGO will provide information fundamental to our understanding of the interaction of gravity in the strong field strength regime. This will include direct studies on black hole normal modes and inertial frame dragging around rotating black holes. The relativistic equations of motion resulting from the post-Newtonian approximation will be detailed through the studies of compact binary systems containing neutron stars and black holes during the final moment of coalescence. Neutron star binary systems will provide information on the neutron star equation of state. LIGO will be able to directly measure the speed of propagation of gravitational waves, and working in conjunction with other interferometers will be able to directly measure the polarization states (instrumental in determining if the general theory of relativity is the correct theory of gravity). Gravitational waves from a compact binary inspiral will provide a new distance measurement method allowing an independent determination of the Hubble constant. Undoubtedly, the most exciting new astrophysics to come out of observations of gravitational waves will be those phenomena that are unexpected and not observable with electromagnetic radiation.

The gravitational wave traverses space-time, producing a cyclic elongation and contraction of bodies in the plane perpendicular to the direction of propagation. There is negligible absorption, scattering or dispersion of the gravitational wave as it propagates. The time evolution of the gravitational wave is analogous to that of electromagnetic waves and is given by

$$
h(\vec{r}, t)=h_{\circ} e^{i(\vec{k} \cdot \vec{r}-\omega t)} .
$$

Like the electromagnetic wave, the gravitational wave can be represented by the superposition of two orthogonal polarizations, $h_{\times}$and $h_{+}$which are illustrated in Figure 2. Gravitational wave emissions from the distribution of massive objects is dominated by the quadrapole moment $Q_{\circ}$ (the dipole moment produces no gravitational radiation). 


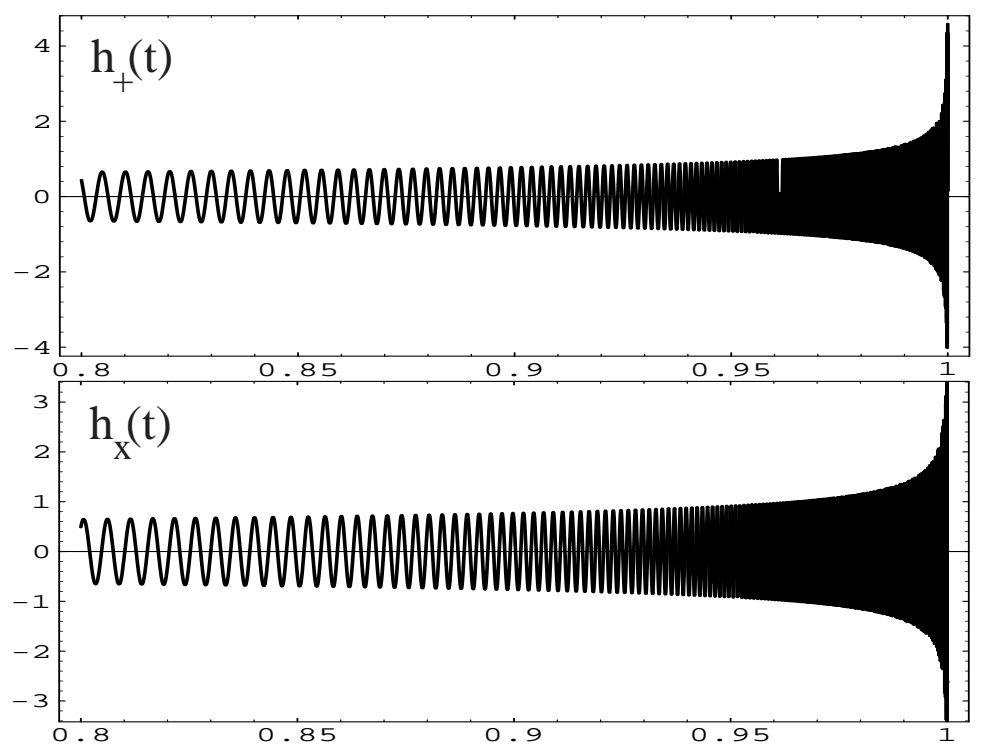

Figure 3: $h_{+}(t)$ and $h_{\times}(t)$ waveforms for final 200 milliseconds of a binary system composed of two 10 solar mass objects with an inclination angle of $30^{\circ}$ at a distance of 10 megaparsecs. The vertical axis is the strain in units of $10^{-20}$.

The amplitude of the gravitational wave $h_{\circ}$ as approximated by the quadrapole moment is

$$
h_{\circ} \simeq \frac{G}{c^{4} r} \frac{d^{2} Q_{\circ}}{d t^{2}} \simeq \frac{G}{c^{4} r} E_{k i n e t i c}^{n s}
$$

where the $E_{\text {kinetic }}^{n s}$ is the kinetic energy resulting from non-spherical internal motions of the source. Consider a typical gravitational wave source located in the Virgo cluster of galaxies, having a distribution of mass on the order of our Sun's mass moving at a few tenths of the speed of light. Such a source would at most produce a strain amplitude on the order of $h_{\circ} \approx 10^{-20}$ and would likely be several orders of magnitude smaller.

Sources of gravitational waves will come from regions of space-time where gravity is relativistic and the distribution of matter is experiencing bulk motions close to the speed of light. Astrophysical candidates for strong gravitational waves most likely to be observed by LIGO include non-axisymmetric supernovae in our own galaxy, non-spherical collapse of a massive star into a black hole, nearby rotating neutron stars with asymmetric mass distributions and the inspiral of compact binary systems such as neutron-neutron, neutron-black hole, and black hole-black hole binaries. Of these the inspiral of compact binary systems is the most understood. To Newtonian order, the inspiralling gravitational waveform is given by

$$
\begin{gathered}
h_{+}(t)=\frac{2 G^{\frac{5}{3}}}{c^{4}}\left(1+\cos ^{2}(\imath)\right) \frac{\mu}{r}(\pi M f)^{\frac{2}{3}} \cos (2 \pi f t) \\
h_{\times}(t)= \pm \frac{4 G^{\frac{5}{3}}}{c^{4}} \cos (\imath) \frac{\mu}{r}(\pi M f)^{\frac{2}{3}} \sin (2 \pi f t)
\end{gathered}
$$

where the polarization axes $\vec{e}_{\times}$and $\vec{e}_{+}$are oriented along the major and minor axes of 
the projection of the orbital plane on the sky, $\imath$ is the angle of inclination of the orbital plane, $M=m_{1}+m_{2}$ is the total mass, $\mu=m_{1} m_{2} / M$ is the reduced mass and the gravitational wave frequency $f$, which is twice the orbital frequency, evolves as a function of time according to

$$
f(t)=\frac{1}{\pi}\left(\frac{c^{3}}{G}\right)^{\frac{5}{8}}\left(\frac{5}{256 \mu M^{\frac{2}{3}}\left(t_{\circ}-t\right)}\right)^{\frac{3}{8}}
$$

where $t_{\circ}$ is the time of coalescence. These waveforms are characterized by a sinusoidal signal that sweeps up in both frequency and in amplitude as a function of time. This "chirp" signal as it is called, is demonstrated in Figure 3 for both polarizations from a pair of 10 solar mass objects at a distance of 10 megaparsecs (Virgo cluster) and with an orbit inclined at $30^{\circ}$ to the source direction.

The Newtonian order waveforms do not provide the needed accuracy to track the phase evolution of the inspiral to a quarter of a cycle over the many thousands of cycles that a typical inspiral will experience while sweeping through the broad band LIGO interferometers. In order to better track the phase evolution of the inspiral, first order corrections to the Newtonian quadrapole radiation, known as the post-Newtonian formulation, were worked out in $1976[4,5]$. However, the post-Newtonian waveforms will not have a sufficiently large fitting factor to be useful as templates in the search for gravitational waves from inspiralling compact binaries $[6,26]$. The gravitational waveforms from inspiralling compact binaries are now known to second post-Newtonian order [7, 24]. At this order it should be possible to accurately track the phase evolution and extract parametric information about the binary system such as the masses, spins, distance and orbital inclination.

3. Principles of detection. The initial LIGO interferometer configuration illustrated in Figure 4, consists of a Michelson interferometer with Fabry-Perot Arm Cavities. The interferometers are designed to detect differential RMS motions between each of the perpendicular arms as small as $10^{-18}$ meters. This corresponds to approximately $10^{-12}$ of the wavelength of the Nd:YAG laser or equivalently, a phase shift measurement of $10^{-9}$ radians. To achieve this level of measurement accuracy, the initial interferometers will incorporate a highly stabilized laser with an input power of 6 Watts at the recycling mirror. Recycling factors of 30 or more will be used to increase the input power to the Fabry-Perot arm cavities. The cavities will have finesses on the order of 100, consistent with the requirement that the light storage time be less than half the period of the gravitational wave to be detected. All optical components contributing to the phase sensitivity of the interferometers will be suspended as pendula and isolated seismically to reduce coupling to thermal and ground motions. The laser wavelength is servo-locked to the average length $\left(L_{1}+L_{2}\right) / 2$ of the interferometer arms. The optical path lengths are maintained by a servo-system specifically to keep the laser light on the photodetector and locked to a particular dark fringe.

This optical configuration requires that four degrees of freedom be controlled by the servo-systems; the differential motion of the cavity $L_{1}-L_{2}$ which is proportional to the gravitational wave signal, the common mode motion of the cavities $L_{1}+L_{2}$, the 


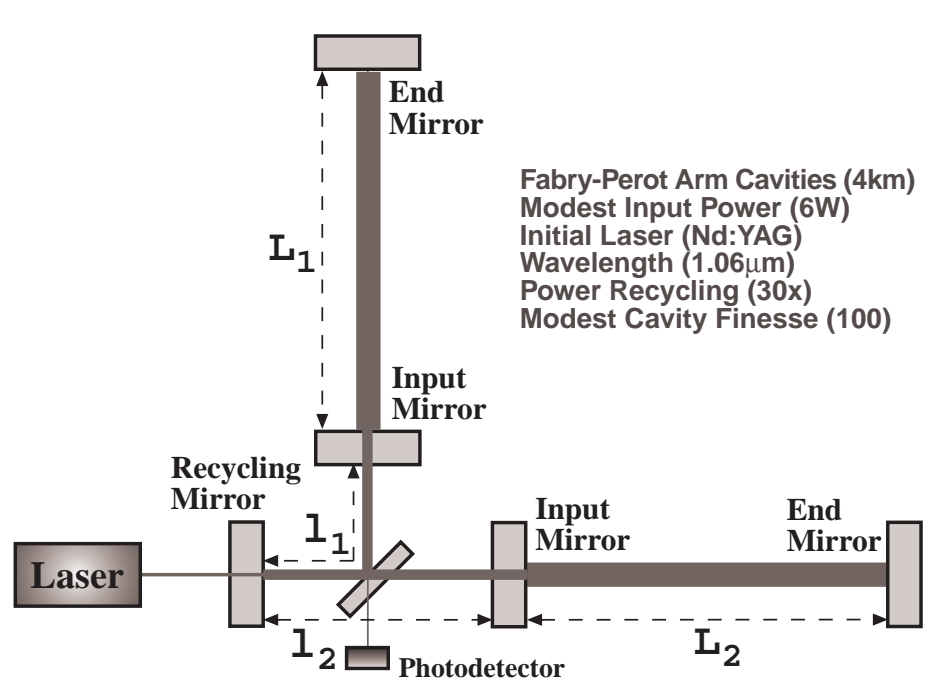

Figure 4: Initial LIGO interferometer configuration

differential motion of the Michelson arms $l_{1}-l_{2}$, and the common mode motion of the Michelson arms $l_{1}+l_{2}$.

In order to reduce the laser amplitude fluctuation effects to below shot noise levels, a modulation scheme is used to shift the measurement to much higher frequencies in the range of $10 \mathrm{MHz}$ using Pockel cells. Several methods of phase detection utilizing modulation schemes have been studied $[8,9,10]$ and continue to be considered for use in LIGO. To maximize the sensitivity to any of the four degrees of freedom, the sideband fields produced by the phase modulation are chosen such that the carrier frequency is in resonance in the Fabry-Perot cavity, while the modulation induced sidebands are not in resonance. Optimization of the interferometer configuration involves studies of the multidimensional coupled system, including the physical characterizations of all the mirrors. Detailed studies using sophisticated simulations software developed on scalable parallel computer architectures are underway.

A gravitational wave couples to the differential mode, $\Delta L=L_{1}-L_{2}$, of the interferometers. The signal $h(t)$ observed at that anti-symmetric port of the interferometer is proportional to this and will depend on the direction to the source relative to the interferometer arms as well as the polarization axes of the gravitational wave by

$$
h(t)=\frac{\Delta L}{L}=F_{+}(\theta, \phi, \psi) h_{+}(t ; \imath, \beta)+F_{\times}(\theta, \phi, \psi) h_{\times}(t ; \imath, \beta)
$$

where $h_{+}(t ; \imath, \beta)$ and $h_{\times}(t ; \imath, \beta)$ are the two polarizations of the gravitational wave as a function of time and source orientation on the sky, and $F_{+}$and $F_{\times}$are the detector beam patterns given by

$$
\begin{aligned}
F_{+}(\theta, \phi, \psi) & =\frac{1}{2}\left(1+\cos ^{2}(\theta)\right) \cos (2 \phi) \cos (2 \psi)-\cos (\theta) \sin (2 \phi) \sin (2 \psi) \\
F_{\times}(\theta, \phi, \psi) & =\frac{1}{2}\left(1+\cos ^{2}(\theta)\right) \cos (2 \phi) \sin (2 \psi)+\cos (\theta) \sin (2 \phi) \sin (2 \psi)
\end{aligned}
$$


where the angles $(\theta, \phi)$ specify the direction from which the gravitational wave is impinging, and the angle $\psi$ specifies the angle the polarization axis is rotated from the constant $\phi$ plane. The magnitude of the beam patterns is always less than or equal to one.

The actual output at the interferometer which results from a gravitational wave producing the strain signal described by Equation 6 is affected by the light storage time of the Fabry-Perot cavities in the arms. A study of this effect results in the complex response $T_{I F O}$ of the interferometer with arms pointing along the $x$ and $y$ axes to a gravitational wave of frequency $f$ to be

$$
\begin{aligned}
T_{I F O}(f)=\frac{\phi(f)}{h_{\circ}(f)}= & \frac{8 \pi \omega_{c} L_{\circ}^{2}}{\lambda c}\left(G_{x x} H\left(f, k_{x}\right)-G_{y y} H\left(f, k_{y}\right)\right) \times \\
& \left(\frac{e^{\frac{i 2 \pi f L_{\circ}}{c}}}{1-2\left(1-\frac{2 \omega_{c} L_{\circ}}{c}\right) e^{\frac{i 2 \pi f L_{\circ}}{c}} \cos \left(\frac{2 \pi f L_{\circ}}{c}\right)+\left(1-\frac{2 \omega_{c} L_{\circ}}{c}\right)^{2} e^{\frac{i 4 \pi f L_{\circ}}{c}}}\right)
\end{aligned}
$$

where $h_{\circ}$ is the strain from the gravitational wave in the plane perpendicular to the direction of motion. The functions $G_{x x}$ and $G_{x x}$ are given by

$$
\begin{aligned}
& G_{x x}(\theta, \phi, \psi)=\cos (2 \psi)\left(\cos ^{2}(\phi)-\sin ^{2}(\phi) \cos ^{2}(\theta)\right)-\sin (2 \psi) \sin (2 \phi) \cos (\theta) \\
& G_{y y}(\theta, \phi, \psi)=\cos (2 \psi)\left(\sin ^{2}(\phi)-\cos ^{2}(\phi) \cos ^{2}(\theta)\right)+\sin (2 \psi) \sin (2 \phi) \cos (\theta)
\end{aligned}
$$

and the functions $H\left(f, k_{x}\right)$ and $H\left(f, k_{y}\right)$ are given by

$$
\begin{aligned}
H\left(f, k_{x}\right) & =\operatorname{sinc}\left(\frac{2 \pi f L_{\circ}}{c}(\sin (\theta) \sin (\phi)-1)\right) e^{\left(\frac{i \pi f L_{\circ}}{c}(\sin (\theta) \sin (\phi)+1)\right)} \\
& +\operatorname{sinc}\left(\frac{2 \pi f L_{\circ}}{c}(\sin (\theta) \sin (\phi)+1)\right) e^{\left(\frac{i \pi f L_{\circ}}{c}(\sin (\theta) \sin (\phi)-1)\right)} \\
H\left(f, k_{y}\right) & =\operatorname{sinc}\left(\frac{2 \pi f L_{\circ}}{c}(\sin (\theta) \cos (\phi)-1)\right) e^{\left(\frac{i \pi f L_{\circ}}{c}(\sin (\theta) \cos (\phi)+1)\right)} \\
& +\operatorname{sinc}\left(\frac{2 \pi f L_{\circ}}{c}(\sin (\theta) \cos (\phi)+1)\right) e^{\left(\frac{i \pi f L_{\circ}}{c}(\sin (\theta) \cos (\phi)-1)\right)}
\end{aligned}
$$

with the constants $L_{\circ}$ being the length of the interferometer arms, and $\omega_{c}$ being the knee frequency of the Fabry-Perot cavity given by

$$
\omega_{c}=\frac{c}{2 L_{\circ}} \frac{1-r_{1} r_{2}}{r_{1} r_{2}}
$$

where $r_{1}$ is the reflectivity of the vertex mirror of the cavity and $r_{2}$ is the reflectivity of the end mirror of the cavity and usually taken to be very close to one.

The response function given by Equation 9 simplifies greatly for gravitational wave frequencies less than $c / 4 \pi L_{\circ}$. For a gravitational strain $h(f)$ given by the Fourier transform of Equation 6, the response for low frequency gravitational waves can be approximated by

$$
T_{I F O}=\frac{\phi(f)}{h(f)} \simeq \frac{4 \pi c}{\lambda \omega_{c}} \frac{1}{\sqrt{1+\left(\frac{2 \pi f}{\omega_{c}}\right)^{2}}} .
$$


4. LIGO noise model. The sensitivity of the LIGO interferometers is limited by irreducible sources of noise. The noise characterization within the interferometer is of two types: Gaussian noise which agrees with the probability distribution of Gaussian statistics, and non-Gaussian noise. The non-Gaussian noise may occur several times per day from events such as strain release in the suspension systems. The only way to remove these non-Gaussian events is through coincidence comparison of each of the signals from the three LIGO interferometers.

Gaussian noise has an extremely fast fall off in probability for increasing noise amplitude. Because of this, the Gaussian noise is unlikely to generate noise bursts and can be characterized by an amplitude spectral density $\tilde{x}(f)$. The signal observed at the photodetector consists of the true gravitational wave strain $h(t)$, plus the Gaussian noise $h_{\text {noise }}(t)$. The amplitude spectral density, $\tilde{x}(f)$ is given by the square root of the power spectral density of $h_{\text {noise }}(t)$. When a gravitational wave having a characteristic strain amplitude $h_{a m p}$ and mean frequency $f_{c}$ is observed by the interferometer for a duration of $n$ cycles, the measured signal to noise ratio will have the following dependency on the noise

$$
\frac{S}{N} \simeq \frac{h_{c}}{h_{r m s}} \simeq \frac{h_{a m p} \sqrt{n}}{\sqrt{f_{c}} \tilde{x}\left(f_{c}\right)}
$$

It is clear from this expression for the signal to noise ratio that the sensitivity and performance of LIGO as an instrument for gravitational wave studies is intimately related to the sources of noise, in particular the Gaussian noise limits this expression. The dominant sources of noise have been studied using the 40 meter prototype interferometer at Caltech and the 5 meter interferometer at MIT. Models have been developed based on this research. The remainder of this section will focus on these Gaussian noise sources that have been modeled, along with their associated parameters, and the expected influence they will have on the initial sensitivity of LIGO.

4.1. Seismic noise. At frequencies below approximately $70 \mathrm{Hertz}$, the noise in the interferometer will be dominated by "seismic noise." This seismic noise originates from the ambient vibrations of the ground due to the geological activity of the Earth, wind forces coupled to trees and buildings, and man-made sources such as traffic, trains, motors and pumps. The ground vibrations couple to the mirrors through the seismic isolation system and the wire suspension which supports the mirrors.

The three tables below give the piece-wise continuous fits to the measured ground motions at the sites used to model the seismic noise in the LIGO interferometers. There

Quiet Hanford Ground Motion

\begin{tabular}{|c|c|}
\hline Frequency Range $\mathbf{( H z})$ & Ground Motion $(\mathrm{m} / \sqrt{\mathrm{Hz}})$ \\
\hline \hline$f<0.1$ & $5.93 \times 10^{-7}$ \\
\hline $0.1 \leq f<0.15$ & $5.93 \times 10^{-4} f^{3}$ \\
\hline $0.15 \leq f<1.0$ & $1.0 \times 10^{-9} f^{-4}$ \\
\hline $1.0 \leq f<8.0$ & $1.0 \times 10^{-9}$ \\
\hline$f \geq 8.0$ & $6.4 \times 10^{-8} f^{-2}$ \\
\hline
\end{tabular}


Noisy Hanford Ground Motion

\begin{tabular}{|c|c|}
\hline Frequency Range $\mathbf{( H z})$ & Ground Motion $(\mathrm{m} / \sqrt{\mathrm{Hz}})$ \\
\hline \hline$f<0.1$ & $5.93 \times 10^{-7}$ \\
\hline $0.1 \leq f<0.15$ & $5.93 \times 10^{-4} f^{3}$ \\
\hline $0.15 \leq f<1.0$ & $1.0 \times 10^{-9} f^{-4}$ \\
\hline $1.0 \leq f<10.0$ & $1.0 \times 10^{-9}$ \\
\hline$f \geq 10.0$ & $1.0 \times 10^{-7} f^{-2}$ \\
\hline
\end{tabular}

Livingston Ground Motion

\begin{tabular}{|c|c|}
\hline Frequency Range $\mathbf{( H z})$ & Ground Motion $(\mathrm{m} / \sqrt{\mathrm{Hz}})$ \\
\hline \hline$f<0.1$ & $1.33 \times 10^{-6}$ \\
\hline $0.1 \leq f<0.15$ & $1.33 \times 10^{-3} f^{3}$ \\
\hline $0.15 \leq f<1.3$ & $2.36 \times 10^{-8} f^{-3}$ \\
\hline $1.3 \leq f<1.5$ & $7.0 \times 10^{-9}$ \\
\hline$f \geq 1.5$ & $1.58 \times 10^{-8} f^{-2}$ \\
\hline
\end{tabular}

are two such fits for Hanford, one representing quiet times when the wind is light and noisy for times when the wind has moderate strength. The Livingston site has less variation in ground motion and has only one fit.

The ground motion is transmitted to the top of the seismic isolation system through four stages composed of compact mass elements on separate legs, separated by vacuum compatible elastomer springs. This design reduces the Q's of internal resonances while maintaining high frequency isolation. In general, how such a system couples ground motion through the system is given by a set of equations of the form

$$
\tilde{X}_{i, t o p}(f)=T_{i j, s t a c k}(f) \cdot \tilde{X}_{j, g r d}(f) .
$$

The seismic isolation stack is nearly cylindrical in shape. Taking this into account, a simplification on the number of degrees of freedom coupled to the ground motion can be fully utilized. The system can then be described by a two dimensional transfer matrix

$$
\left(\begin{array}{c}
\tilde{x}_{t o p}(f) \\
\tilde{z}_{t o p}(f)
\end{array}\right)=\left(\begin{array}{ll}
T_{x x}(f) & T_{x z}(f) \\
T_{z x}(f) & T_{z z}(f)
\end{array}\right)\left(\begin{array}{c}
\tilde{x}_{g r d}(f) \\
\tilde{z}_{g r d}(f)
\end{array}\right) .
$$

Horizontal motion is also coupled to tilt in this simplified model. The resultant horizontal motion at the stack suspension point that results from transmission along the down-tube of length $L_{D T}$ with stack support legs out a distance $R$ from the center is given by

$$
\tilde{x}_{p i t c h}(f)=\frac{2 L_{D T}}{R} T_{z x}(f) \cdot \tilde{x}_{g r d}(f) .
$$

Using these simplifications the two dimensional transfer function has been measured from a prototype seismic isolation stack located at MIT [11] and appropriately scaled to the LIGO configuration. The transmission curves for $T_{x x}, T_{x z}, T_{z x}$ and $T_{z z}$, are shown in Figure 5 . 
LIGO Stack Transfer Functions

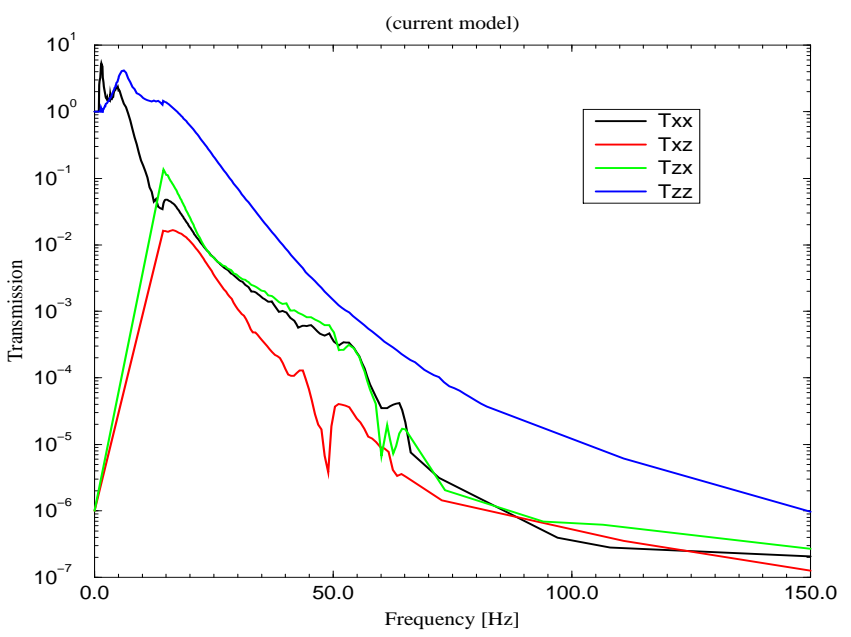

Figure 5: LIGO Seismic Isolation System transfer functions for the simplified two dimensional model. Transfer functions are based on measurements made on a prototype which have been scaled to the LIGO design.

Transmission from the top of the stack to the stack suspension point at the base of the down-tube for the $(x, z)$ components is given by

$$
\begin{gathered}
\tilde{x}_{\text {suspension }}(f)=\sqrt{\tilde{x}_{\text {top }}^{2}(f)+\tilde{x}_{\text {pitch }}^{2}(f)} \\
\tilde{z}_{\text {suspension }}(f)=\tilde{z}_{\text {top }}(f) .
\end{gathered}
$$

The motion found at the stack suspension point is further translated into motion at the mirrors through the pendulum's horizontal and vertical transmission. Neglecting cross coupling terms, the motion at the mirror surface is given by

$$
\begin{gathered}
\tilde{x}_{\text {mirror }}(f)=T_{\text {horizontal pendulum }}(f) \cdot \tilde{x}_{\text {suspension }}(f) \\
\tilde{z}_{\text {mirror }}(f)=T_{\text {vertical pendulum }}(f) \cdot \tilde{z}_{\text {suspension }}(f)
\end{gathered}
$$

where the horizontal pendulum transfer function $T_{\text {horizontal pendulum }}(f)$ and the vertical pendulum transfer function $T_{\text {vertical pendulum }}(f)$ are given by

$$
\begin{gathered}
T_{\text {horizontal pendulum }}(f)=\frac{Q_{H} f_{H}^{2}\left(f_{H}^{2}-f^{2}\right)-i Q_{H} f_{H}^{3} f}{Q_{H}^{2}\left(f_{H}^{2}-f^{2}\right)^{2}-f_{H}^{2} f^{2}} \\
T_{\text {vertical pendulum }}(f)=\frac{Q_{V} f_{V}^{2}\left(f_{V}^{2}-f^{2}\right)-i Q_{V} f_{V}^{3} f}{Q_{V}^{2}\left(f_{V}^{2}-f^{2}\right)^{2}-f_{V}^{2} f^{2}} .
\end{gathered}
$$

Due to the curvature of the Earth over the 4 kilometer arm lengths, the local surface horizontal and the laser beam will differ by a small angle $\delta \theta$. This provides a coupling between the local vertical motion of the mirror and displacements along the beam axis. When this coupling is taken into effect, the total displacement along the beam from the 
ground motion transmitted to the mirrors is given by

$$
\tilde{x}_{\text {beam }}(f)=\sqrt{\tilde{x}_{\text {mirror }}^{2}+\left(\delta \theta \cdot \tilde{z}_{\text {mirror }}\right)^{2}} .
$$

The seismic noise found in the interferometer will be given by the root square sum of the displacement from each mirror's motion along the beam. The resultant seismic noise for the interferometer is given by

$$
\tilde{x}_{\text {Seismic }}(f)=\sqrt{\sum_{i=1}^{4} \tilde{x}_{\text {beam }, i}^{2}(f)} \simeq 2 \tilde{x}_{\text {beam }}(f) .
$$

The seismic noise model combines these expressions with the measured transfer functions and ground motion. The model also includes a set of free parameters which have been measured in the laboratory or specified in the LIGO design. These parameters are given in the following table.

\begin{tabular}{|c|c|c|c|}
\hline Parameter & Symbol & Value & Units \\
\hline \hline horizontal pendulum frequency & $f_{H}$ & 0.744 & Hertz \\
\hline horizontal pendulum quality factor & $Q_{H}$ & $3.33 \times 10^{5}$ & factor \\
\hline vertical pendulum frequency & $f_{V}$ & 13.0 & Hertz \\
\hline vertical pendulum quality factor & $Q_{V}$ & 333.3 & factor \\
\hline local beam angle & $\delta \theta$ & $3.1 \times 10^{-4}$ & radians \\
\hline down tube length & $L_{D T}$ & 0.90 & meters \\
\hline lever arm length & $R$ & 0.63 & meters \\
\hline
\end{tabular}

4.2. Thermal noise. At frequencies above approximately 70 Hertz and below approximately $200 \mathrm{~Hz}$, the noise in the interferometer will be dominated by thermal noise sources. LIGO will operate at room temperatures $\left(\sim 295^{\circ} \mathrm{K}\right)$. The dominant sources of this noise are the thermally induced off-resonance vibrations of the mirrors, suspensions and the top plate of the seismic isolation system. Thermally excited resonance vibrations of the mirrors and suspensions do appear at higher frequencies where shot noise dominates. The influence of high $\mathrm{Q}$ thermally induced resonances in the mirrors and suspensions will be important for understanding the broadband performance of the interferometer and are included in the models for thermal noise.

4.2.1. Top plate thermal noise. Thermal excitations of the last stage of the seismic isolations system induce motions at the suspension point which are transferred to the mirrors. This particular source of thermal noise is not limiting in the initial LIGO detector, but is important for the understanding of the sources of sensitivity limitations in more advanced detector designs.

There are two possible damping mechanisms [12] in the top plate, velocity damping and internal damping. Velocity or viscous damping is likely to give a higher noise floor in the region dominated by thermal noise sources, since the power spectrum above resonance for this type of damping falls off as $1 / f^{4}$. The expression used for the power spectral 
density of the motion in the top plate is given by

$$
\tilde{x}_{\text {velocity }}^{2}=\frac{4 k_{B} T f_{\text {vel }}}{8 \pi^{3} m\left(Q_{\text {vel }}\left(f_{\text {vel }}^{2}-f^{2}\right)^{2}+f_{\text {vel }}^{2} f^{2}\right)} .
$$

For internal or structural damping, the power spectrum above resonance falls off as $1 / f^{5}$. The expression for the power spectral density of the top plate motion is given by

$$
\tilde{x}_{\text {internal }}^{2}=\frac{4 k_{B} T f_{i n t}^{2} \phi_{i n t}}{8 \pi^{3} m f\left(\left(f_{i n t}^{2}-f^{2}\right)^{2}+f_{i n t}^{4} \phi_{i n t}^{2}\right)} .
$$

Both of these thermal excitations couple to the mirror motion through the pendulum transfer function. The vertical thermal excitations which couple to the mirror motion along the beam axis through the Earth's curvature are several orders of magnitude less than the seismic induced vertical motions at the top plate and therefore will be neglected in this model. The horizontal pendulum transfer function is given by Equation 24. When taken together, the motion along the beam from the thermal modes of the top plate gives

$$
\tilde{x}_{\text {beam }}(f)=T_{\text {horizontal pendulum }}(f) \cdot \tilde{x}_{\left\{\frac{\text { velocity }}{\text { internal }}\right\}}(f)
$$

where $\left\{\frac{\text { velocity }}{\text { internal }}\right\}$ represents the type of damping mechanism used to determine the amplitude spectral density and in general is selected to be velocity damping in the model. Each seismic isolation stack's top plate contributes to the noise in the interferometer. The sum over each stack's top plate gives

$$
\tilde{x}_{\text {Topplate }}(f)=\sqrt{\sum_{i=1}^{4} \tilde{x}_{\text {beam }, i}^{2}(f)} \simeq 2 \tilde{x}_{\text {beam }}(f) .
$$

The top plate thermal noise model combines these expressions with the following set of parameters which have been measured in the laboratory or specified in the LIGO design.

\begin{tabular}{|c|c|c|c|}
\hline Parameter & Symbol & Value & Units \\
\hline \hline temperature & $T$ & 295.37 & Kelvin \\
\hline top plate mass & $m$ & 250.0 & kilograms \\
\hline velocity damped frequency & $f_{\text {vel }}$ & 4.0 & Hertz \\
\hline velocity damped quality factor & $Q_{\text {vel }}$ & 3.0 & factor \\
\hline internal damped frequency & $f_{\text {int }}$ & 6.0 & Hertz \\
\hline internal damped loss function & $\phi_{\text {int }}$ & 0.333 & factor \\
\hline horizontal pendulum frequency & $f_{H}$ & 0.744 & Hertz \\
\hline horizontal pendulum quality factor & $Q_{H}$ & $3.33 \times 10^{5}$ & factor \\
\hline
\end{tabular}

4.2.2. Pendulum and violin mode thermal noise. A major source of noise for the initial LIGO in this frequency range is the thermally induced vibration of the pendulum. Determining the damping of the pendulum at frequencies far from the pendulum resonance is difficult. In order to estimate the pendulum's damping, the violin quality factor $Q_{V}$ is used. The pendulum thermal noise [13] associated with a single suspended mirror 
is given by

$$
\tilde{x}_{\text {pendulum }}^{2}(f)=\frac{4 k_{B} T \phi_{P} f_{P}^{2}}{8 \pi^{3} m f\left(\left(f_{P}^{2}-f^{2}\right)^{2}+\phi_{P}^{2} f_{P}^{4}\right)}
$$

where the pendulum mode loss function is related to the violin loss function by the relationship

$$
\phi_{P}=\sum_{i=1}^{N_{\text {wires }}} \frac{\phi_{V}}{2 N_{\text {wires }}} .
$$

LIGO will use a single loop suspension to support the mirrors. Thus in equation 33, $N_{\text {wires }}$ is two. The relationship between the pendulum loss function and the violin loss function therefore reduces to

$$
\phi_{V}=2 \phi_{P} .
$$

The thermal noise from the violin modes are also important in modeling the thermal noise of LIGO. The violin thermal noise associated with a single suspended mirror is given by the sum over all harmonics of the fundamental frequency $f_{V}$,

$$
\tilde{x}_{\text {violin }}^{2}(f)=\sum_{k=1}^{N_{\text {modes }}} \frac{4 k_{B} T \phi_{V} f_{P}^{2}}{8 \pi^{3} m f\left(\left(\left(k f_{V}\right)^{2}-f^{2}\right)^{2}+\phi_{V}^{2}\left(k f_{V}\right)^{4}\right)} .
$$

The combined thermal noise from the pendulum and violin modes associated with a single loop suspension is given by the root square sum of equations 32 and 35

$$
\tilde{x}_{\text {loop }}(f)=\sqrt{\tilde{x}_{\text {pendulum }}^{2}(f)+\tilde{x}_{\text {violin }}^{2}(f)} .
$$

The total thermal noise found in the interferometer from the pendulum and violin modes of all four suspensions supporting the mirrors is given by the sum

$$
\tilde{x}_{\text {wires }}(f)=\sqrt{\sum_{i=1}^{4} \tilde{x}_{\text {loop }, i}^{2}(f)} \simeq 2 \tilde{x}_{\text {loop }}(f) .
$$

The suspension's pendulum and violin mode thermal noise model combine these expressions with measured or LIGO specified parameters that are listed in the following table.

\begin{tabular}{|c|c|c|c|}
\hline Parameter & Symbol & Value & Units \\
\hline \hline temperature & $T$ & 295.37 & Kelvin \\
\hline mirror mass & $m$ & 10.8 & kilograms \\
\hline pendulum frequency & $f_{P}$ & 0.744 & Hertz \\
\hline pendulum loss function & $\phi_{P}$ & $3.0 \times 10^{-6}$ & loss \\
\hline fundamental violin frequency & $f_{v}$ & 376.0 & Hertz \\
\hline violin loss function & $\phi_{V}$ & $6.0 \times 10^{-6}$ & Hertz \\
\hline number of harmonics & $N_{\text {modes }}$ & 32 & number \\
\hline
\end{tabular}

4.2.3. Vertical spring mode thermal noise. Another source of noise in the suspension is the vertical spring mode. Thermally induced vibrations of this mode do not contribute 
significantly to the total thermal noise of the initial interferometers but the characterization of this noise is important in the design of LIGO. The thermally driven vertical motion is given by

$$
\tilde{z}^{2}(f)=\frac{4 k_{B} T f_{V}^{2} \phi_{V}}{8 \pi^{3} m f_{V}\left(\left(f_{V}^{2}-f^{2}\right)^{2}+f_{V}^{4} \phi_{V}^{2}\right)} .
$$

The thermally induced vertical motion $\tilde{z}(f)$ along the pendulum wires couples to the mirror displacement as a result of the curvature of the Earth

$$
\tilde{x}_{\text {beam }}=\delta \theta \cdot \tilde{z}(f) .
$$

Combining this motion along the beam axis from all four mirrors gives

$$
\tilde{x}_{\text {vertical spring }}=\sqrt{\sum_{i=1}^{4} \tilde{x}_{\text {beam }, i}^{2}(f)} \simeq 2 \tilde{x}_{\text {beam }}(f) .
$$

The suspension's vertical spring mode thermal noise model combines these expressions with measured or LIGO specified parameters listed in the table below.

\begin{tabular}{|c|c|c|c|}
\hline Parameter & Symbol & Value & Units \\
\hline \hline temperature & $T$ & 295.37 & Kelvin \\
\hline mirror mass & $m$ & 10.8 & kilograms \\
\hline vertical pendulum frequency & $f_{V}$ & 13.0 & Hertz \\
\hline vertical pendulum loss function & $\phi_{V}$ & $3.0 \times 10^{-3}$ & loss \\
\hline local beam angle & $\delta \theta$ & $3.1 \times 10^{-4}$ & radians \\
\hline
\end{tabular}

4.2.4. Pitch and yaw mode thermal noise. Two remaining sources of thermal noise within the suspension system are incorporated within the initial LIGO noise model. These are the pitch and yaw modes of the mirrors. For small amplitudes, these modes contribute to the motion of the mirrors along the beam in proportion to the degree of off-centering, $(\Delta y, \Delta z)$, of the beamspot on the mirror. The power spectral density for the pitch mode is given by

$$
\tilde{x}_{\text {beam }}^{2}(f)=\frac{4 k_{B} T \Delta z^{2} \phi_{P} f_{P}^{2}}{8 \pi^{3} I_{P} f\left(\left(f_{P}^{2}-f^{2}\right)^{2}+\phi_{P}^{2} f_{P}^{4}\right)}
$$

which when combined for all four mirrors results in the total pitch mode thermal noise being

$$
\tilde{x}_{\text {pitch }}(f)=\sqrt{\sum_{i=1}^{4} \tilde{x}_{\text {beam }, i}^{2}(f)} \simeq 2 \tilde{x}_{\text {beam }}(f) .
$$

The suspension's pitch mode thermal noise model combines these expressions with measured or LIGO specified parameters listed in the table below. 


\begin{tabular}{|c|c|c|c|}
\hline Parameter & Symbol & Value & Units \\
\hline \hline temperature & $T$ & 295.37 & Kelvin \\
\hline mirror pitch moment of inertia & $I_{P}$ & $5.12 \times 10^{-2}$ & $\mathrm{~kg} / \mathrm{m}^{2}$ \\
\hline beam centering deviation & $\Delta z$ & $1.0 \times 10^{-3}$ & meters \\
\hline pitch mode frequency & $f_{P}$ & 0.6 & Hertz \\
\hline pitch mode loss function & $\phi_{P}$ & $8.0 \times 10^{-4}$ & loss \\
\hline
\end{tabular}

Similarly, the power spectral density for the yaw mode is given by

$$
\tilde{x}_{\text {beam }}^{2}(f)=\frac{4 k_{B} T \Delta y^{2} \phi_{Y} f_{Y}^{2}}{8 \pi^{3} I_{Y} f\left(\left(f_{Y}^{2}-f^{2}\right)^{2}+\phi_{Y}^{2} f_{Y}^{4}\right)}
$$

which, when combined for all four mirrors results in the total yaw mode thermal noise being

$$
\tilde{x}_{\text {yaw }}(f)=\sqrt{\sum_{i=1}^{4} \tilde{x}_{\text {beam }, i}^{2}(f)} \simeq 2 \tilde{x}_{\text {beam }}(f) .
$$

The suspension's yaw mode thermal noise model combines these expressions with measured or LIGO specified parameters listed in the table below.

\begin{tabular}{|c|c|c|c|}
\hline Parameter & Symbol & Value & Units \\
\hline \hline temperature & $T$ & 295.37 & Kelvin \\
\hline mirror yaw moment of inertia & $I_{Y}$ & $5.12 \times 10^{-2}$ & $\mathrm{~kg} / \mathrm{m}^{2}$ \\
\hline beam centering deviation & $\Delta y$ & $1.0 \times 10^{-3}$ & meters \\
\hline yaw mode frequency & $f_{Y}$ & 0.5 & Hertz \\
\hline yaw mode loss function & $\phi_{Y}$ & $2.5 \times 10^{-4}$ & loss \\
\hline
\end{tabular}

4.2.5. Mirror internal mode thermal noise. Thermally excited vibrations of the mirror's internal modes contribute significantly to the thermal noise within this frequency region [14]. In the model used to calculate this source of thermal noise, the mirrors are treated as three-dimensional bodies [15] and the cumulative effects from the modes is tracked in order to accurately reach convergence. The coupling of the mirror modes to the optical modes is carefully treated allowing a more detailed estimate of the motion along the optical path length affecting the interferometer sensitivity.

The power spectral density associated with a particular internal mode of the mirror is given by

$$
\tilde{x}_{n}^{2}(f)=\frac{4 k_{B} T}{8 \pi^{3} \alpha_{n} m f}\left(\frac{f \phi_{n}(f)}{\left(f_{n}^{2}-f^{2}\right)^{2}+f_{n}^{4} \phi_{n}(f)}\right)
$$

where $\alpha_{n}$ is the effective mass coefficient which characterizes the contribution to the thermal noise for the $n$-th mode

$$
\alpha_{n}=\frac{\frac{1}{2} k_{B} T}{\frac{1}{2} m \omega_{n} \Delta l_{n}^{2}} .
$$

The effective mass coefficient $\alpha_{n}$ is a measure of the coupling of the optical mode $\psi_{00}$ (only the $\mathrm{TEM}_{00}$ mode is considered since other modes do not resonate in the Fabry-Perot 


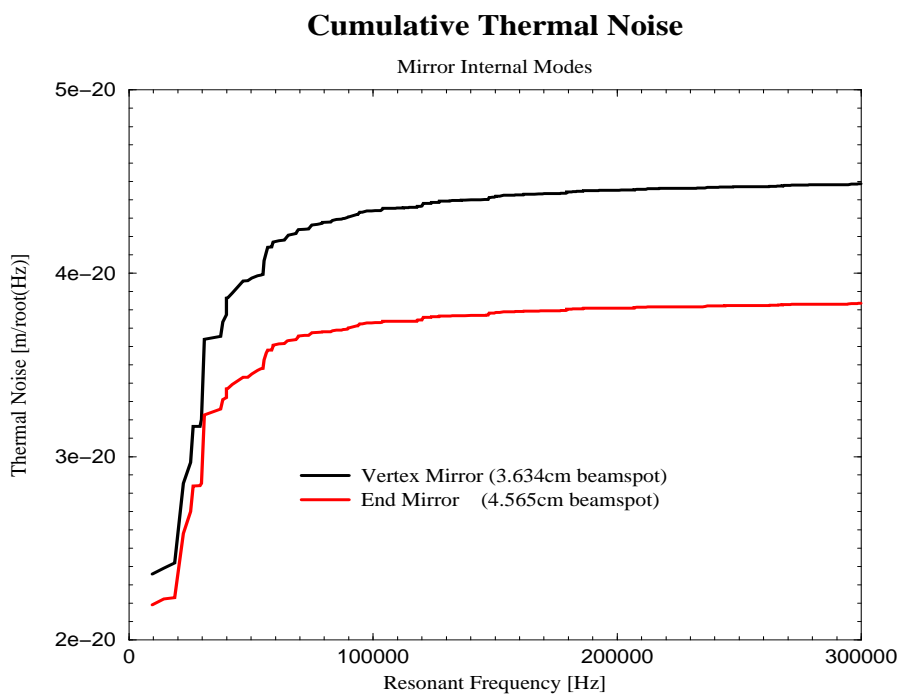

Figure 6: The cumulative contribution to the internal thermal noise of each of the 4 kilometer interferometer mirrors. The mirror dimensions are 12.5 centimeters in radius by 10 centimeters in length. The beamspot sizes for the vertex and end mirrors are 3.634 and 4.565 centimeters respectively. The cumulative summing was carried out to a maximum resonant frequency of $3.0 \times 10^{5}$ Hertz.

cavity) to the mirror's internal mode surface displacement $u_{n}$, by the integral

$$
\Delta l_{n}=\frac{\lambda}{2 \pi} \oint_{S} \psi_{00}^{*} \psi_{00}\left(\vec{k} \cdot \vec{u}_{n}\right) d \sigma
$$

where the phase shift experienced by the optical mode upon reflection off the mirror is given by

$$
\psi_{00}(\rho, \theta, z)=\psi_{00}(z) e^{i 2 \vec{k} \cdot \vec{u}_{n}(\rho, \theta)} .
$$

Expanding this for the case of small $\left|\vec{k} \cdot \vec{u}_{n}\right|$ leads to the simplification

$$
\psi_{00}(\rho, \theta, z) \simeq \psi_{00}(z)\left(1+i 2 \vec{k} \cdot \vec{u}_{n}(\rho, \theta)-2\left(\vec{k} \cdot \vec{u}_{n}(\rho, \theta)\right)^{2}\right) .
$$

Losses in the mirror substrate are assumed to be independent of frequency. This is in agreement with current experimental results in fused silica [16]. All modes are taken to have the same loss function, hence the quality factor is given by

$$
\phi_{n}(f)=\frac{1}{Q_{n}} \simeq \text { constant } .
$$

The modes are summed until a reasonable convergence is reached in the cumulative thermal noise as illustrated in Figure 6

$$
\tilde{x}_{\text {modes }}(f)=\sqrt{\sum_{n} \tilde{x}_{n}^{2}(f)}
$$




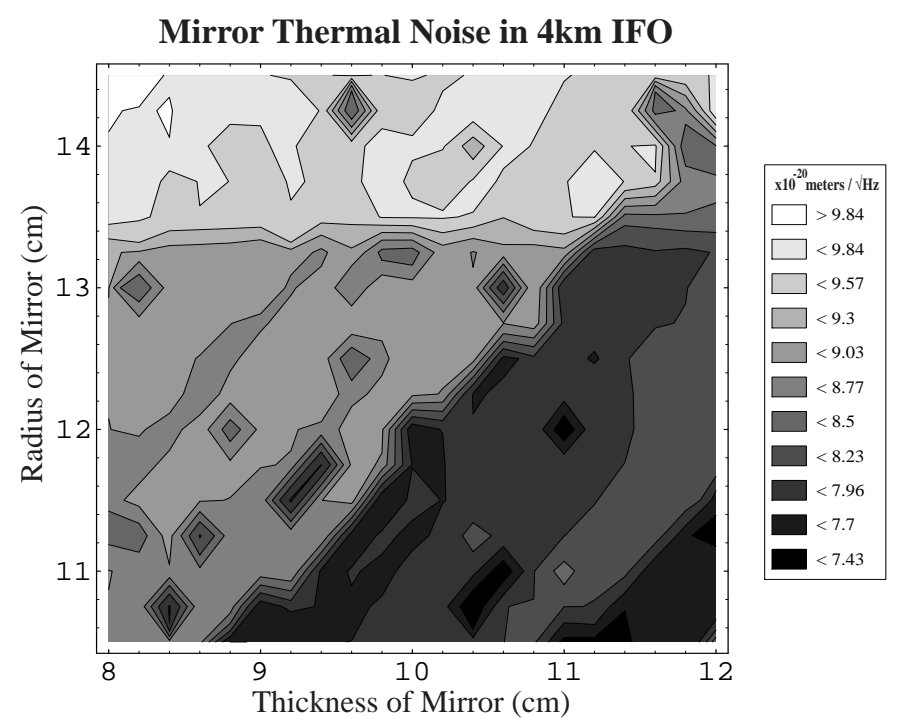

Figure 7: The contours illustrate the dependency of the mirror's internal mode thermal noise on the cylinder dimensions at 100 Hertz for the 4 kilometer interferometer's mirrors.

Combining all four mirrors results in the total mirror internal mode thermal noise

$$
\tilde{x}_{\text {Mirror }}(f)=\sqrt{\sum_{i=1}^{4} \tilde{x}_{\text {modes }, i}^{2}(f)} \simeq 2 \tilde{x}_{\text {modes }}(f) .
$$

This model has been applied to study dependency of the thermal noise on the dimensions of the mirror. This is extremely computationally intensive, requiring thousands of node hours on a Sun network. The results of the calculations are shown in Figure 7 and suggest the optimum dimensions for the mirror. The mirror internal mode thermal noise model combines these models with measurements and LIGO specified parameters listed in the following table.

\begin{tabular}{|c|c|c|c|}
\hline Parameter & Symbol & Value & Units \\
\hline \hline temperature & $T$ & 295.37 & Kelvin \\
\hline mirror density & $\rho$ & 2200.0 & $\mathrm{~kg} / \mathrm{m}^{3}$ \\
\hline mirror radius & $R_{\text {mirror }}$ & 0.125 & meters \\
\hline mirror length & $L$ & 0.10 & meters \\
\hline mirror loss function & $\phi$ & $4.0 \times 10^{-7}$ & loss \\
\hline minimum mode frequency & $f_{\min }$ & 1000.0 & Hertz \\
\hline maximum mode frequency & $f_{\max }$ & 250000.0 & Hertz \\
\hline number of radial series terms & $N_{R}$ & 30 & number \\
\hline number of axial series terms & $N_{Z}$ & 30 & number \\
\hline vertex beamspot radius & $R_{v}$ & 0.03634 & meters \\
\hline end beamspot radius & $R_{e}$ & 0.04565 & meters \\
\hline
\end{tabular}


4.3. Shot noise. At frequencies above approximately 200 Hertz the noise in the interferometer is dominated by shot noise. This noise source is the result of the random nature of the photon arrival times at the photodetector. The highly sensitive optical arrangement to be used in LIGO implements phase modulation. The modulation scheme shifts the gravitational wave signal to higher frequencies, away from technical noises, and then after demodulation the signal is recovered free of those technical noises [17]. These schemes also provide additional signals for use in monitoring and controlling the interferometer's optical path length degrees of freedom. However, these schemes modify the standard shot noise formula, which assume constant light power. When the effects of the LIGO modulation scheme and power recycling are taken into effect, the equivalent to displacement amplitude spectral density from the phase noise induced by the shot noise if given by [18]

$$
\tilde{x}_{S h o t}(f)=\left(\frac{\lambda \sqrt{3 E_{S B}^{2}+E_{D C}^{2}}}{4 \pi E_{R C} E_{S B}} \frac{\left(1-r_{1} r_{2}\right)^{2}}{\left(1-r_{1}^{2}-L_{1}\right) r_{2}}\right) \sqrt{1+\left(\frac{2 \pi f}{\omega_{c}}\right)^{2}}
$$

where the $E_{S B}^{2}$ is the electric field power in the sidebands produced by the phase modulation

$$
E_{S B}^{2}=\frac{\lambda \eta P_{i n} J_{1}^{2}(\Gamma)}{h c},
$$

$E_{R C}^{2}$ is the electric field power in the recycling cavity

$$
E_{R C}^{2}=\frac{\lambda \eta P_{i n} G J_{0}^{2}(\Gamma)}{h c}
$$

and $E_{D C}^{2}$ is the total DC power on the photodetector from asymmetries in the arm cavity as well as other sources of stray power such as higher order spatial modes

$$
E_{D C}^{2}=P_{\text {stray }} \text {. }
$$

The Fabry-Perot cavity pole frequency $\omega_{c}$ is a function of the cavity length and reflectivities of the mirrors and is given by

$$
\omega_{c}=\frac{c}{2 L_{\circ}} \frac{1-r_{1} r_{2}}{r_{1} r_{2}} .
$$

The shot noise model outlined above is combined with parameters listed in the table below which are based on LIGO design specifications.

\begin{tabular}{|c|c|c|c|}
\hline Parameter & Symbol & Value & Units \\
\hline \hline input laser power & $P_{\text {in }}$ & 6.0 & watts \\
\hline recycling gain & $G$ & 30.0 & factor \\
\hline modulation depth & $\Gamma$ & 0.45 & fraction \\
\hline photodiode efficiency & $\eta$ & 0.80 & fraction \\
\hline vertex reflectivity & $r_{1}$ & 0.9849 & amplitude \\
\hline end reflectivity & $r_{2}$ & 0.999995 & amplitude \\
\hline vertex losses & $L_{1}$ & 50.0 & ppm \\
\hline wavelength & $\lambda$ & $1.064 \times 10^{-6}$ & meters \\
\hline arm-length & $L_{\circ}$ & 4000.0 & meters \\
\hline
\end{tabular}


4.4. Radiation pressure noise. Photons in the laser light induce a second source of noise in the interferometer known as radiation pressure noise. This noise arises from the forces imparted on the mirrors as statistically different numbers of photons reflect off the mirrors in the two arms. The amplitude spectral density for this process is given by

$$
\tilde{x}_{R P}(f)=\frac{\mathcal{N}}{m f^{2}} \sqrt{\frac{2 \hbar G P_{i n}}{\pi^{3} c \lambda}}
$$

The number of characteristic bounces of the light $\mathcal{N}$ appearing in this expression is related to the finesse $\mathcal{F}$ of the Fabry-Perot cavity,

through the relationship

$$
\mathcal{F}=\frac{\pi \sqrt{r_{1} r_{2}}}{1-r_{1} r_{2}}
$$

$$
\mathcal{N}=\frac{2 \mathcal{F}}{\pi}=\frac{2 \sqrt{r_{1} r_{2}}}{1-r_{1} r_{2}} .
$$

The radiation pressure noise model outlined above is combined with parameters listed in the table below which are based on LIGO design specifications.

\begin{tabular}{|c|c|c|c|}
\hline Parameter & Symbol & Value & Units \\
\hline \hline mirror mass & $m$ & 10.8 & kilograms \\
\hline input laser power & $P_{i n}$ & 6.0 & watts \\
\hline recycling gain & $G$ & 30.0 & factor \\
\hline wavelength & $\lambda$ & $1.064 \times 10^{-6}$ & meters \\
\hline vertex reflectivity & $r_{1}$ & 0.9849 & amplitude \\
\hline end reflectivity & $r_{2}$ & 0.999995 & amplitude \\
\hline
\end{tabular}

4.5. Residual gas phase noise. The LIGO interferometer will operate at pressures on the order of $10^{-9}$ Torr. This effectively isolates the mirrors and suspension from effects such as acoustic coupling and mechanical damping. But statistical fluctuations in the effective index of refraction of the residual gas within the beam tube resulting from variations in the column density seen by the laser beam can limit the strain sensitivity. At the expected partial pressures, this will not be a major source of noise for the initial LIGO but it will place limits on the sensitivity achievable for advanced LIGO interferometers of the future.

The total amplitude spectral density from the residual gas phase noise is given by the root square sum of the contributions from each gas species found within the beam tube

$$
\tilde{x}_{R G}(f)=\sqrt{\sum_{i}^{n}{\tilde{x_{i}}}^{2}(f) .}
$$

Each gas species has a contribution to the above expression which depends on the partial pressure $P$, polarizability $\alpha$ and mass $m$ of the gas molecule [19]. The contribution is also dependent on the shape of beam waist $w(z)$ along the beam axis

$$
{\tilde{x_{i}}}^{2}(f)=\frac{8 \rho_{i}\left(2 \pi \alpha_{i}\right)^{2}}{v_{\circ i}} \int_{z_{1}}^{z_{2}} \frac{e^{-\left(\frac{2 \pi f w(z)}{v_{\circ} i}\right)}}{w(z)} d z
$$


The location of the minimum cross section of the beam is given by $z_{1}$, with the beam extending along the beam axis from $z_{1}$ to $z_{2}$ in these coordinates

$$
z_{1}=-L_{\circ} \frac{g_{2}\left(1-g_{1}\right)}{g_{1}+g_{2}-2 g_{1} g_{2}}, \quad z_{2}=z_{1}+L_{\circ},
$$

where $g_{1}$ and $g_{2}$ are functions of the radii of curvature for the vertex and end mirrors of the cavity

$$
g_{1}=1-\frac{L_{\circ}}{R_{1}}, \quad g_{2}=1-\frac{L_{\circ}}{R_{2}} .
$$

The minimum cross section for the beam is found using the expression

$$
w_{\circ}=\sqrt{\frac{\lambda L_{\circ}}{\pi} \frac{\sqrt{g_{1} g_{2}\left(1-g_{1} g_{2}\right)}}{\left(g_{1}+g_{2}-2 g_{1} g_{2}\right)}}
$$

and the cross section at any point $z$ along the beam is given by

$$
w(z)=w_{\circ} \sqrt{\left(\frac{\lambda z}{\pi w_{\circ}^{2}}\right)^{2}+1} .
$$

The gas species' most probable speed $v_{\circ}$ depends on the mass and temperature of the molecule

$$
v_{\circ i}=\sqrt{\frac{2 k_{B} T}{m_{i}}}
$$

and the number density for the gas species depends on the pressure and temperature

$$
\rho_{i}=\frac{P_{i}}{R T} .
$$

The set of parameters relevant to the residual gas phase noise for LIGO are given in the table below. A factor of 133.3224 is used to convert partial pressures $P_{i}$ from Torr to $\mathrm{N} / \mathrm{m}^{2}$ before using the number density in the amplitude spectral density formula.

\begin{tabular}{|c|c|c|c|}
\hline Parameter & Symbol & Value & Units \\
\hline \hline armlength & $L_{\circ}$ & 4000.0 & meters \\
\hline wavelength & $\lambda$ & $1.064 \times 10^{-6}$ & meters \\
\hline temperature & $T$ & 295.37 & Kelvin \\
\hline vertex mirror curvature & $R_{1}$ & 14558.0 & meters \\
\hline end mirror curvature & $R_{2}$ & 7402.0 & meters \\
\hline
\end{tabular}

The current model also uses parameters for eight of the most prevalent gas species being considered in the calculation. The pressures listed in the last column are representative of expected values for LIGO. 


\begin{tabular}{|c|c|c|c|}
\hline Molecule & $\mathbf{m}(\mathrm{amu})$ & $\alpha\left(\mathrm{m}^{3}\right)$ & $\mathbf{P}($ Torr $)$ \\
\hline \hline hydrogen & 2 & $7.4 \times 10^{-19}$ & $5.0 \times 10^{-9}$ \\
\hline water & 18 & $1.4 \times 10^{-18}$ & $<1.0 \times 10^{-9}$ \\
\hline nitrogen & 28 & $1.6 \times 10^{-18}$ & $<1.0 \times 10^{-9}$ \\
\hline oxygen & 32 & $1.6 \times 10^{-18}$ & $<1.0 \times 10^{-9}$ \\
\hline carbon monoxide & 28 & $1.8 \times 10^{-18}$ & $<1.0 \times 10^{-9}$ \\
\hline carbon dioxide & 44 & $2.38 \times 10^{-18}$ & $<1.0 \times 10^{-9}$ \\
\hline methane & 16 & $2.36 \times 10^{-18}$ & $<1.0 \times 10^{-9}$ \\
\hline hydrocarbon & $X$ & $\alpha_{H_{2}}(0.74+0.137 X)$ & $\ll 1.0 \times 10^{-9}$ \\
\hline
\end{tabular}

4.6. Seismic gravity gradient noise. The ambient ground motions near the interferometers induce density fluctuations which give rise to fluctuating gravitational forces on the mirrors. These in turn constitute a source of noise in the interferometer know as seismic gravity gradient noise. The model for this noise source is based on the theory of Rayleigh and Love waves, which are the dominant form of seismic waves in the frequency range of interest to LIGO [20]. Unlike the seismic noise sources which can in principle be isolated from the mirrors with a sufficiently advanced seismic isolation system, the gravity gradient noise sources act at a distance through the gravitational force. Hence, even though it is not a major noise source for the initial LIGO, it does play a role at low frequencies to limit sensitivity for advanced LIGO interferometers.

The ground motion (given in the discussion on Seismic Noise) is transmitted to the mirror by the gravity gradient transfer function $T_{G G}$

$$
\tilde{x}_{G G}(f)=T_{G G}(f) \tilde{X}_{\text {grd }} .
$$

The actual form of this transfer function depends on the theory of Rayleigh and Love waves and on the geological details of the sites chosen for LIGO. Because of the differences in the geology of between the Hanford and Livingston sites, two unique transfer functions are used by the model.

4.6.1. Livingston transfer function. The geological survey of the Livingston site reveals a nearly homogeneous clay-like material down to depths of greater than 10 meters. Under these conditions, the theory allows the site to be treated as a single homogeneous isotropically elastic medium bounded above by vacuum (air). The transfer function for this site is given by

$$
T_{L A}(f)=\frac{2(1-q)}{1-\frac{2 q s}{1+s^{2}}} \frac{G \rho \gamma\left(\frac{2 \pi f l_{\circ}}{c_{R}}\right)}{\sqrt{\left(f^{2}-f_{\circ}^{2}\right)^{2}+\frac{f^{2}}{\tau_{\circ}^{2}}}}
$$

where the function $\gamma$ is given by

$$
\gamma(\nu)=\sqrt{1+\frac{1}{2 \pi} \int_{0}^{2 \pi} \cos (\phi) \sin (\phi) \cos \left(\nu \frac{\cos (\phi)+\sin (\phi)}{\sqrt{2}}\right) d \phi} .
$$

The dimensionless parameters $q$ and $s$ represent the ratio of the vertical e-folding rate to horizontal wave number for the longitudinal and transverse components of the Rayleigh 
waves in the ground

$$
q=\sqrt{1-\frac{c_{R}^{2}}{c_{L}^{2}}}, \quad s=\sqrt{1-\frac{c_{R}^{2}}{c_{T}^{2}}}
$$

and the parameter $\tau_{\circ}$ is the damping time of the horizontal pendulum mode of the mirror suspension system

$$
\tau_{\circ}=\frac{Q_{\circ}}{\pi f_{\circ}} .
$$

The value for the parameters entering into the expressions for the Livingston transfer function $T_{L A}$ are given in the following table and are based on LIGO design specifications and geotechnical data from the Livingston site.

\begin{tabular}{|c|c|c|c|}
\hline Parameter & Symbol & Value & Units \\
\hline \hline pendulum frequency & $f_{\circ}$ & 0.744 & Hertz \\
\hline pendulum quality factor & $Q_{\circ}$ & $3.33 \times 10^{5}$ & factor \\
\hline vertex mirror separation & $l_{\circ}$ & 4.1 & meters \\
\hline ground density & $\rho$ & 2000.0 & $\mathrm{~kg} / \mathrm{m}^{3}$ \\
\hline P-wave sound speed & $c_{L}$ & 500.0 & $\mathrm{~m} / \mathrm{s}$ \\
\hline Raleigh sound speed & $c_{R}$ & 188.0 & $\mathrm{~m} / \mathrm{s}$ \\
\hline S-Wave sound speed & $c_{T}$ & 200.0 & $\mathrm{~m} / \mathrm{s}$ \\
\hline
\end{tabular}

4.6.2. Hanford transfer function. The geological survey of the Hanford site reveals cemented soils down to depths of approximately 200 meters. Sound speed measurements taken from bore holes indicate a two-layer stratification near the surface. The top layer extends to a depth of roughly 5 meters, and the second layer extends from there to a depth of over 20 meters Under these conditions, the theory allows the site to be treated as two homogeneous isotropic elastic media with a common boundary at the depth $D \sim 5$ meters, unbounded below the interface and bounded above by vacuum (air). The transfer function for this site is given by

$$
\begin{gathered}
T_{W A}(f)=\frac{2\left(1-q_{1}\right)}{1-\frac{2 q_{1} s_{1}}{1+s_{1}^{2}}} \frac{G \rho_{1} \gamma\left(\frac{2 \pi f l_{\circ}}{c_{R}}\right)}{\sqrt{\left(f^{2}-f_{\circ}^{2}\right)^{2}+\frac{f^{2}}{\tau_{\circ}^{2}}}} \times \\
\left(1+e^{-\left(q_{1}+1\right) k D}+\frac{\rho_{2}}{\rho_{1}} \frac{1-q_{2}}{1-q_{1}}\left(\frac{q_{1} s_{2}-1}{q_{2} s_{2}-1} e^{-\left(q_{1}+1\right) k D}-\frac{2 e^{-\left(q_{1}+1\right) k D} q_{1}(s 2-s 1)}{\left(q_{2} s_{2}-1\right)\left(s_{1}^{2}+1\right)}\right)\right)
\end{gathered}
$$

where the function $\gamma$ is given by

$$
\gamma(\nu)=\sqrt{1+\frac{1}{2 \pi} \int_{0}^{2 \pi} \cos (\phi) \sin (\phi) \cos \left(\nu \frac{\cos (\phi)+\sin (\phi)}{\sqrt{2}}\right) d \phi} .
$$

The dimensionless parameters $q_{1}$ and $s_{1}$ represent the ratio of the vertical e-folding rate to horizontal wave number for the longitudinal and transverse components of the Love waves in the top layer

$$
q_{1}=\sqrt{1-\frac{c_{R}^{2}}{c_{L 1}^{2}}}, \quad s_{1}=\sqrt{1-\frac{c_{R}^{2}}{c_{T 1}^{2}}}
$$


and as before, the parameter $\tau_{\circ}$ is the damping time of the horizontal pendulum mode of the mirror suspension system

$$
\tau_{\circ}=\frac{Q_{\circ}}{\pi f_{\circ}} .
$$

The value of $q_{2}$ and $s_{2}$ must be solved in a consistent manner to guarantee continuity of the stress across the boundary layer found at the depth $z=D$ at the Hanford site. This is achieved by having $q_{2}$ and $s_{2}$ satisfy the following equations for the given values of $q_{1}$ and $s_{1}$

$$
\begin{gathered}
2 q_{1}\left(e^{-q_{1} k D}-e^{-s_{1} k D}\right)=\frac{\mu_{2}}{\mu_{1}}\left(2 q_{2} F_{1}+\left(s_{2}^{2}+1\right) \frac{F_{2}}{s_{2}}\right) \\
e^{-q_{1} k D}\left(\frac{K_{1}}{\mu_{1}}\left(1-q_{1}^{2}\right)-\frac{2}{3}\left(2 q_{1}^{2}+1\right)\right)+4 \frac{q_{1} s_{1}}{s_{1}^{2}+1} e^{-s_{1} k D} \\
=F_{1}\left(\frac{K_{2}}{\mu_{1}}\left(1-q_{2}^{2}\right)-\frac{2 \mu_{2}}{3 \mu_{1}}\left(2 q_{2}^{2}+1\right)\right)-2 \frac{\mu_{2}}{\mu_{1}} F_{2}
\end{gathered}
$$

where the constants $\mu_{1}, \mu_{2}, K_{1}$ and $K_{2}$ depend on the geological properties of the Hanford site and are given by

$$
\begin{aligned}
\mu_{1}=c_{T 1}^{2} \rho_{1}, & \mu_{2}=c_{T 2}^{2} \rho_{1} \\
K_{1}=c_{L 1}^{2} \rho_{1}-\frac{4}{3} \mu_{1}, & K_{2}=c_{L 2}^{2} \rho_{2}-\frac{4}{3} \mu_{2},
\end{aligned}
$$

the Love wave's horizontal wave number for both the top and bottom layers is given by

$$
k=\frac{2 \pi f}{c_{R}}
$$

and the remaining functional simplifications used to solve $q_{2}$ and $s_{2}$ above are given by

$$
\begin{aligned}
& F_{1}=\frac{1}{q_{2} s_{2}-1}\left(\left(q_{1} s_{1}-1\right) e^{-q_{1} k D}-\frac{2 q_{1}\left(s_{2}-s_{1}\right)}{s_{1}^{2}+1} e^{-s_{1} k D}\right) \\
& F_{2}=\frac{s_{2}}{q_{2} s_{2}-1}\left(\left(q_{2}-q_{1}\right) e^{-q_{1} k D}-\frac{2 q_{1}\left(q_{2} s_{1}-1\right)}{s_{1}^{2}+1} e^{-s_{1} k D}\right) .
\end{aligned}
$$

The value for the parameters entering into the expressions for the Hanford transfer function $T_{W A}$ are given in the following table and are based on LIGO design specifications and geotechnical data from the Hanford site.

\begin{tabular}{|c|c|c|c|}
\hline Parameter & Symbol & Value & Units \\
\hline \hline pendulum frequency & $f_{\circ}$ & 0.744 & Hertz \\
\hline pendulum quality factor & $Q_{\circ}$ & $3.33 \times 10^{5}$ & factor \\
\hline vertex mirror separation & $l_{\circ}$ & 4.1 & meters \\
\hline upper ground density & $\rho_{1}$ & 2000.0 & $\mathrm{~kg} / \mathrm{m}^{3}$ \\
\hline lower ground density & $\rho_{2}$ & 2000.0 & $\mathrm{~kg} / \mathrm{m}^{3}$ \\
\hline upper P-wave sound speed & $c_{L 1}$ & 600.0 & $\mathrm{~m} / \mathrm{s}$ \\
\hline lower P-wave sound speed & $c_{L 1}$ & 1550.0 & $\mathrm{~m} / \mathrm{s}$ \\
\hline Raleigh sound speed & $c_{R}$ & 253.8 & $\mathrm{~m} / \mathrm{s}$ \\
\hline upper S-Wave sound speed & $c_{T 1}$ & 270.0 & $\mathrm{~m} / \mathrm{s}$ \\
\hline lower S-Wave sound speed & $c_{T 1}$ & 420.0 & $\mathrm{~m} / \mathrm{s}$ \\
\hline boundary depth & $D$ & 5.0 & $\mathrm{~meters}$ \\
\hline
\end{tabular}


4.7. Quantum limit. The standard quantum limit for LIGO illustrates the fundamental limitations to measurement imposed by quantum mechanics and the uncertainty principle. The standard quantum limit is not a limiting noise source in the initial LIGO interferometers. However, it does set a fundamental limit on the sensitivity achievable in gravitational wave detectors possessing this technical design.

Determination of the standard quantum limit hinges on optimization of the total optical readout noise. The total power in optical readout noise in the interferometer is given by the sum of the power in the shot noise with the power in the radiation pressure noise. The shot noise decreases with power while the radiation pressure noise increases with power

$$
\tilde{x}_{\text {optical }}(f)=\sqrt{\tilde{x}_{\text {Shot }}^{2}(f)+\tilde{x}_{R P}^{2}(f)} .
$$

Because of this, it is possible to find a minimum in the optical readout noise. This minimum optical readout noise can be found by choosing the power $P_{i n}$ to satisfy, at any particular frequency $\bar{f}$, the relationship

$$
\tilde{x}_{\text {Shot }}^{2}(\bar{f})=\tilde{x}_{R P}^{2}(\bar{f}) .
$$

Solving this equation will result in the optimal input power, which is given by a relationship which is functionally dependent on the frequency

$$
P_{\text {optimal }}(f)=\frac{\pi \lambda c m f^{2}}{2 \mathcal{N}^{2}} .
$$

When this $P_{\text {optimal }}$ is substituted into the equation for $\tilde{x}_{\text {optical }}(f)$, the result is the standard quantum limit. It expresses the fundamental quantum mechanical limit to measurement

$$
\tilde{x}_{Q L}(f)=\frac{1}{\pi f} \sqrt{\frac{2 \hbar}{m}} .
$$

The interesting feature of the standard quantum limit is that it only depends upon a single parameter, the mass of the mirrors.

\begin{tabular}{|c|c|c|c|}
\hline Parameter & Symbol & Value & Units \\
\hline \hline mirror mass & $m$ & 10.8 & kilograms \\
\hline
\end{tabular}

4.8. Summary of noise models. The noise sources outlined in this section have been incorporated into a software package, which runs under the AVS environment. Each noise model exists as a separate module in this environment. All modules use a common database to access the required parameters used to characterize the noise sources in the 4 kilometer interferometers that LIGO is building in Hanford and Livingston. A sophisticated adaptive spacing algorithm is used in all models to guarantee that all details associated with high $Q$ features in the noise sources are accurately represented. The result of running this LIGO noise software package is plotted in Figure 8. The figure includes the root square sum of all the sources (red curve) as well as the noise sensitivity goal set by LIGO (black curve). 


\section{Initial LIGO Noise Curves}

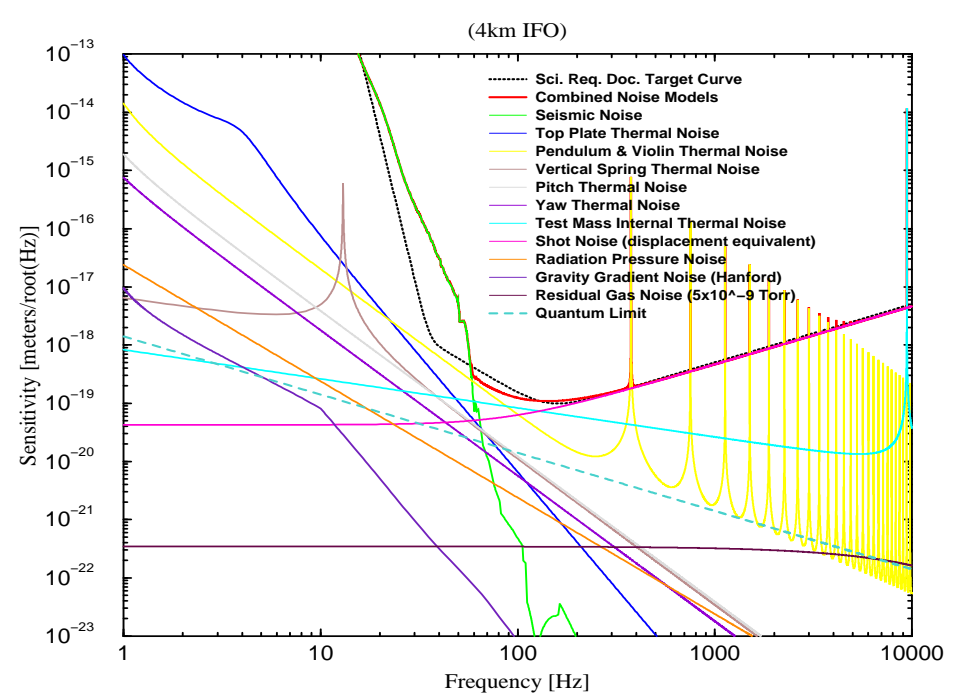

Figure 8: Summary of initial LIGO noise models for the 4 kilometer interferometer.

\section{Initial LIGO Noise Sources}

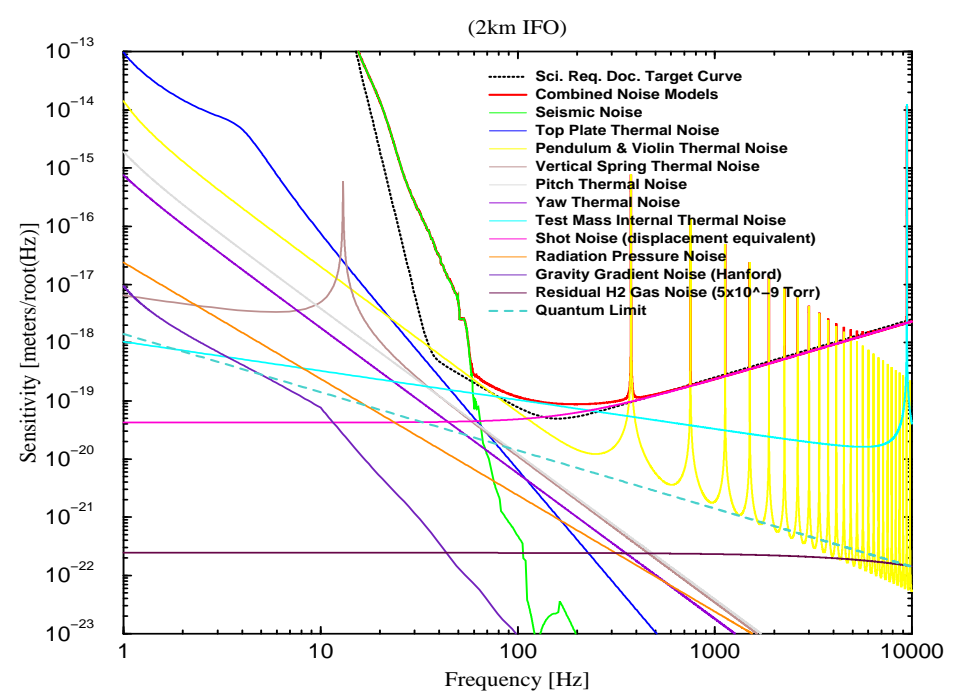

Figure 9: Summary of initial LIGO noise models for the 2 kilometer interferometer.

The Hanford site will also include a half length 2 kilometer interferometer to provide added confidence requirements to any gravitational wave signals detected. A half dozen parameter differ in the proposed design of the 2 kilometer interferometer. These are listed in the following table. 


\begin{tabular}{|c|c|c|c|c|}
\hline Parameter & Symbol & 4km IFO & 2km IFO & Units \\
\hline \hline arm-length & $L_{\circ}$ & 4000.0 & 2000.0 & meters \\
\hline vertex beamspot radius & $R_{v}$ & 0.03634 & 0.0288 & meters \\
\hline end beamspot radius & $R_{e}$ & 0.04565 & 0.0288 & meters \\
\hline vertex mirror curvature & $r_{1}$ & 14558.0 & 4732.0 & meters \\
\hline end mirror curvature & $r_{2}$ & 7402.0 & 4732.0 & meters \\
\hline vertex mirror separation & $l_{\circ}$ & 4.1 & 8.2 & meters \\
\hline
\end{tabular}

Using these parameters, the LIGO noise software package produces the set of plots shown in Figure 9 for the 2 kilometer interferometer at Hanford. The red curve for the root square sum reveals that the thermal noise in the 2 kilometer interferometer limits the sensitivity in the region around 100 to 200 Hertz to above the 4 kilometer design sensitivity shown by the black curve. A design sensitivity has not been specified for the 2 kilometer interferometer at this time.

5. Implementation. Land at both sites have been cleared. The Hanford site has been graded and the beam tube slabs have been poured. The building construction contract has just been awarded as of this writing in the summer of 1996. Final grading of the Livingston site is currently underway. Fabrication of the beam tube has begun. Research and development continues at both Caltech and MIT, where design and implementation of the initial LIGO detector is progressing in the areas of recycling, wavefront sensing, lock acquisition and others.

5.1. Facilities. The two sites will each have a corner stations complex (see Figure 10) to house the lasers, vertex interferometer components and vacuum equipment (LVEA). The complex will include an Operations and Support Building (OSB) to house the control rooms, labs and office space. Each site will also include two end stations to house the vacuum equipment and optics associated with the end mirrors. The Hanford site will have two mid-stations to house the vacuum equipment and optics associated with the 2 kilometer interferometer's end mirrors.

The corner, mid and end stations will be connected by the welded stainless steel beam tube (see Figure 11) in which the long arms of the Fabry-Perot cavities will be contained. The beam tube will be covered by a beam tube enclosure built from cast concrete in 3 meter long sections. The enclosure will have a height of 2.7 meters and a width of 4 meters. It will provide protection to the beam tube from wind and stray bullets from hunters and give some degree of passive temperature stabilization.

The facilities have been designed to reduce environmental noise from vibrations and acoustic noise. The designs also address the necessary shielding and isolation of electromagnetic noise sources and electrical grounding. The sites for LIGO have been selected based on their quiet ambient ground motion and remote locations.

5.2. Beam tube and vacuum system. The beam tube, illustrated in Figure 11, is being built in modular lengths of 2 kilometers each, with approximately 100 kilometers of welding. The diameter of the beam tube is 1.22 meters. Approximately 300 internal 


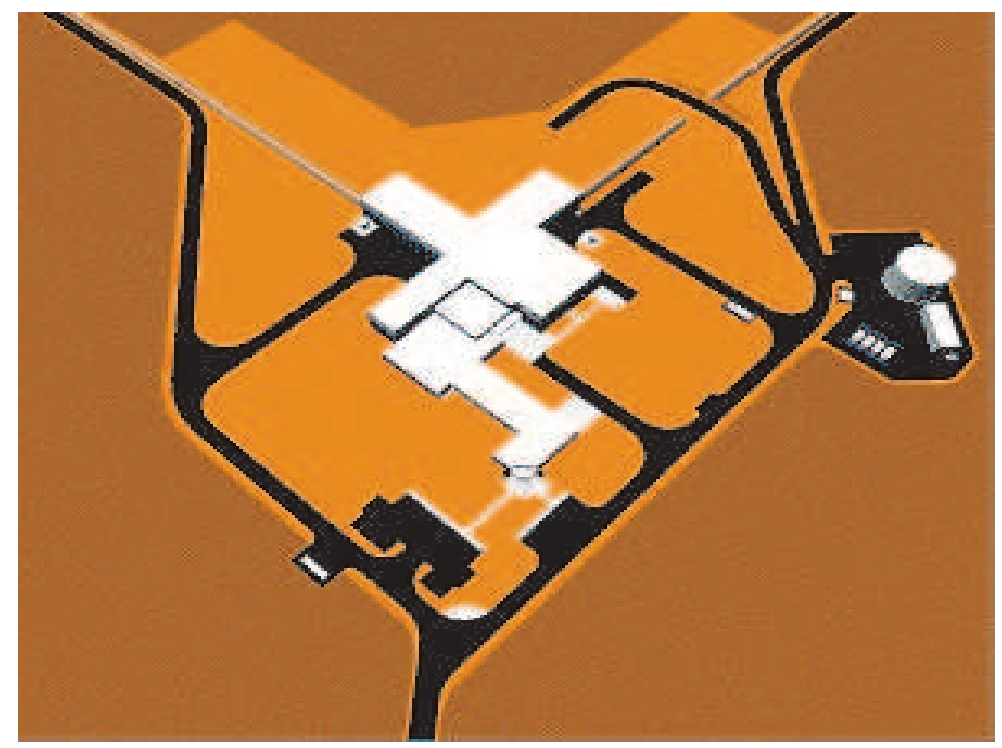

Figure 10: The Corner Station Complex showing the Laser and Vacuum Equipment Area (LVEA), the Operations and Support Building (OSB) and the beam tubes extending out of the field of view.

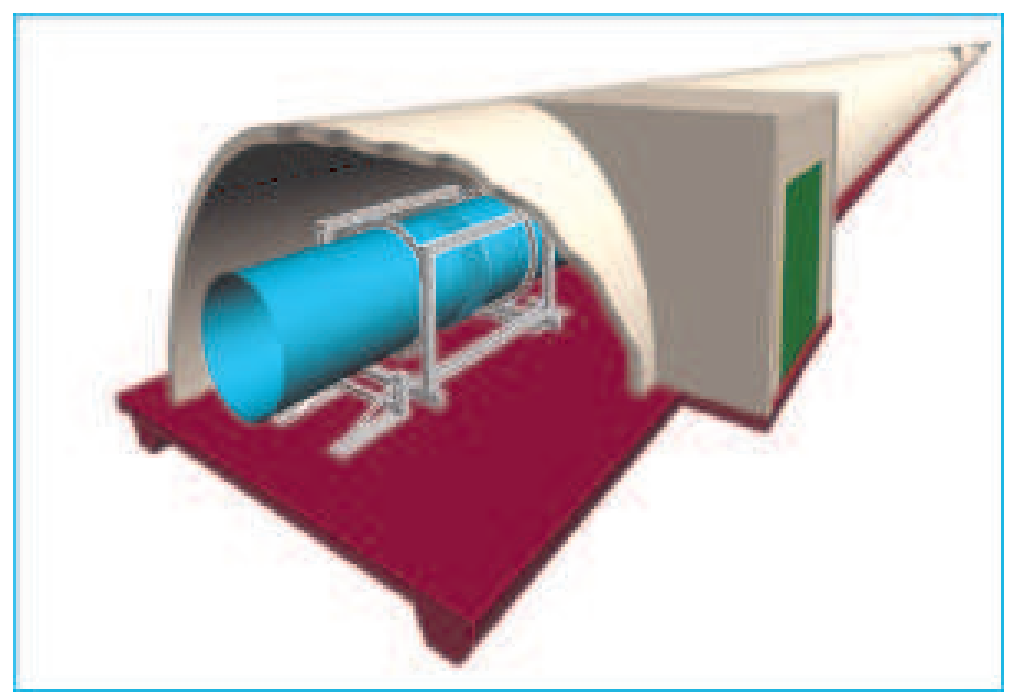

Figure 11: Cut-away view of the LIGO beam tube supported by a slab and covered by the beam tube enclosure.

baffles will be used in each arm to reduce scattered light phase noise. The baffles are being coated with a "black glass" similar to coatings used in household ovens.

Air leakage rates will be less than $10^{-9}$ atm cc/second. All residual species of gas will have partial pressures of less than $10^{-9}$ Torr in the advanced LIGO. Leak tests made 
on the prototype produced results of less than $10^{-11} \mathrm{~atm}$ cc/second. Quality control and cleanliness during the fabrication and integration are being systematically pursued in order to guarantee a long lifetime with the specified leak rates.

Together with the vacuum system, roughly $2 \times 10^{5}$ meters $^{3}$ of ultra-high vacuum will provide a clear aperture for the interferometer, as well as an optically clean environment for the precision optics. The chambers of the vacuum system will be large enough to allow easy access to interferometer components during installation and maintenance periods.

5.3. Control and data system. The LIGO Control and Data System (CDS) is an advanced control and data system using state of the art hardware components and network architecture. The system will manage unique aspects of LIGO such as control and monitoring, interferometer diagnostics and data acquisition. Timing for the CDS will be derived from Global Positioning System (GPS). Hardware will be based on standard VME crates and integrated modules.

\begin{tabular}{|c|c|c|c|c|c|}
\hline System & $\mathbf{2 ~ H z}$ & $\mathbf{2 5 6} \mathbf{~ H z}$ & $\mathbf{2 0 4 8} \mathbf{~ H z}$ & $\mathbf{1 6 3 8 4} \mathbf{~ H z}$ & Total \\
\hline \hline Suspension & 120 & 90 & 30 & 60 & 300 \\
\hline Prestabilized Laser & 20 & 10 & 5 & 8 & 43 \\
\hline Mode Cleaner & 30 & 20 & 10 & 20 & 80 \\
\hline Input Optics & 20 & 15 & 5 & 10 & 50 \\
\hline IFO Readout & 20 & 15 & 0 & 30 & 65 \\
\hline Alignment & 20 & 15 & 0 & 0 & 35 \\
\hline Channels/IFO & 230 & 165 & 50 & 128 & 573 \\
\hline KBytes/sec/IFO & 0.9 & 84.5 & 204.8 & 4194.3 & 4484.5 \\
\hline \hline Auxiliary & 0 & 200 & 10 & 30 & 240 \\
\hline Housekeeping & 300 & 50 & 20 & 0 & 370 \\
\hline Channels/Site & 300 & 250 & 30 & 30 & 610 \\
\hline KBytes/sec/Site & 1.2 & 128 & 122.9 & 983.0 & 1235.1 \\
\hline
\end{tabular}

The CDS system will control and monitor the interferometer, the vacuum system and the physical environment. Data sample rates, shown in the table above, will range from 2 sample per second up to $16 \mathrm{~K}$ samples per second. Data collection from each interferometer is estimated to be 6 megabytes per second and will collect data from approximately 1500 channels per interferometer. The data will be recorded to tape using a "framebuilder" to write the data in frames of a compatible format with other gravitational wave research groups. The data stored in these frames will be the basis of both on-line and off-line data analyses.

5.4. Detector. Development of the initial detector system has been the responsibility of the current research and development programs at Caltech and MIT. Ongoing research utilizes three prototype interferometers, the 40 meter interferometer at Caltech and the phase noise and fixed mass interferometers at MIT. These projects will continue to pursue technological advances after the initial LIGO installation for incorporation into future advanced interferometer designs. 


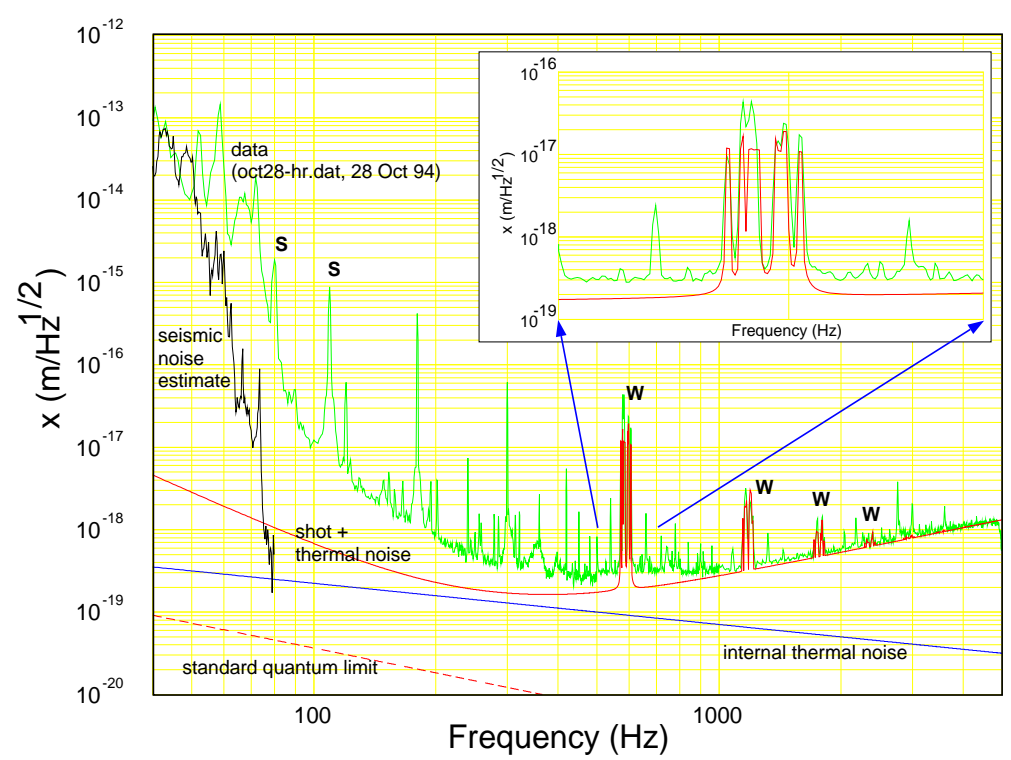

Figure 12: Displacement sensitivity of the 40 meter interferometer at Caltech in comparison to the predicted performance from the noise models.

At Caltech the 40 meter interferometer has been instrumental in the study of noise and performance. Figure 12 demonstrates the comparison of the measured sensitivity with expectation based on theory. The 40 meter interferometer is also the testbed for verification of LIGO subsystems such as the pre-stabilized laser and prototypes of the CDS systems. The current configuration is one of optical recombination and will soon evolve to a recycled configuration. A prototype data acquisition system will be added near the end of 1996.

The phase noise interferometer at MIT is working to achieve the phase sensitivity required by LIGO of less than $10^{-10}$ radians $/ \sqrt{\mathrm{Hz}}$ with a recycling power on the order of 100 Watts. The phase noise interferometer recently achieved a recycling gain of 450 . The next step will be to convert to an Nd:YAG laser. This interferometer is also being used to test an active vibration isolations system.

The fixed mass interferometer at MIT is being set up to perform initial tests of the frequency-shifted, subcarrier length sensing control system. It will also be used to develop a wavefront sensor to maintain optimal alignment in a locked interferometer.

6. Data analysis. The LIGO interferometers will begin continuous collection of data at roughly the beginning of the new millennium. Gravitational wave detection will be the core of LIGO operations. While new and unexpected discoveries will undoubtedly be a part of LIGO's legacy, the search for gravitational waves of a known form as predicted by the general theory of relativity and astrophysics will be the driving mode of data analysis in the initial phase of LIGO.

In anticipation of this phase, a great deal of intellectual energy is being focused to understand the challenges of detecting and characterizing the data collected by LIGO. 
Major sources of gravitational waves fall into four astrophysically distinct categories. The implications to LIGO data analysis from each of these categories are unique and summarized below.

6.1. Supernovae. Supernovae can potentially generate gravitational waves detectable by the initial LIGO interferometers through several mechanisms, even in the case of spherical collapse. The neutron star produced in a supernova is likely to be convectively unstable and "boil" vigorously in a manner which radiates gravitationally during the initial $\sim 0.1$ seconds of its existence [21]. It is estimated that something on the order of 10 cycles of gravitational waves will be generated by this boiling with a frequency of $\sim 100$ Hertz with a gravitational wave amplitude of

$$
h \simeq 3 \times 10^{-22} \frac{30 \mathrm{kpc}}{r} .
$$

The initial LIGO would only be able to detect such amplitudes within the local group of galaxies, leading to event rates of roughly one every ten years.

If the supernova progenitor is rotating then the collapsing core will likely be centrifugally flattened, enabling gravitational radiation during the collapse. If the core's angular momentum is small then the evolution is likely to be axisymmetric and the amplitude of the gravitational waves will be quite small, possibly having a characteristic amplitude of

$$
h_{c} \simeq 3 \times 10^{-21} \frac{30 \mathrm{kpc}}{r}
$$

with a frequency between 200 and 1000 Hertz which will precede the boiling phase by a fraction of a second. Since the number of wave cycles will be quite small during the collapse, the initial LIGO interferometers will only be able to observe such signals from within the local group of galaxies with event rates comparable to those from the convective boiling.

If the angular momentum of the supernova core is large, then dynamical and secular instabilities may force a non-axisymmetric core to form. It is conceivable that the core could break up into two or more pieces. In this case the gravitational wave signal could be nearly as strong as in the case of a coalescing compact binary (discussed below). If the core is centrifugally hung up with a radius of approximately 100 kilometers, as may be the case if the progenitor core is a white dwarf spun up by accretion, then the instability will produce a bar or bifurcation which radiates at approximately 100 Hertz. If the excess angular momentum goes into the production of gravitational waves, then the waveforms will increase in frequency (chirp) with a characteristic amplitude of roughly half that found in a neutron star - neutron star binary coalescence. However, the angular momentum will probably go into the production of hydrodynamic waves, reducing the amplitude of the gravitational waves from these events.

The other possibility in the case of large angular momentum is for the core to become centrifugally hung up at a radius just larger than the final neutron star $(\sim 20$ kilometers $)$. Numerical simulations of this case [22] reveal that after a short phase of emission at a frequency of approximately $1000 \mathrm{Hertz}$, the core reaches a secular instability in which the frequency decreases to about $10 \mathrm{Hertz}$ having a characteristic amplitude only slightly less than in the case of chirp waveform from the previous case of centrifugal hang up at 
a radius of approximately 100 kilometers. Fits to the numerical simulations [23] give

$$
\begin{aligned}
& h_{+}(t)=A(f)\left(\frac{1+\cos (\imath)}{2}\right) \cos (\Phi(t)) \\
& h_{\times}(t)=A(f) \cos (\imath) \sin (\Phi(t))
\end{aligned}
$$

where the amplitude $A(f)$ is given by

$$
A(f)=4.6 \times 10^{-22} \frac{M^{2}}{R}\left(\frac{30 \mathrm{Mpc}}{r}\right) B\left(f_{\max }\right)\left(\frac{f}{f_{\max }}\right)^{2.1} \sqrt{1-\frac{f}{f_{\max }}}
$$

and where $M$ is the mass of the neutron star in units of 1.4 solar masses, $R$ is the radius of the neutron star in units of 10 kilometers and $B\left(f_{\max }\right)$ is

where

$$
B\left(f_{\max }\right)= \begin{cases}\left(\bar{f}_{\max } / 1756\right)^{2.7} & \text { for } \bar{f}_{\max }<500 H z \\ \left(\bar{f}_{\max } / 1525\right)^{3} & \text { for } \bar{f}_{\max }>330 H z\end{cases}
$$

$$
\bar{f}_{\text {max }}=26.7 \sqrt{\frac{R^{3}}{M}} f_{\text {max }} .
$$

The phase evolution for this gravitational waveform is given by

$$
\Phi(t)=2 \pi \int f(t) d t
$$

where the frequency is calculated from the number of wave cycles per logarithmic frequency

$$
\frac{f^{2}}{\dot{f}}=-\sqrt{\left|\frac{d N}{d \ln f}\right|}=\frac{0.03\left(\frac{R^{4}}{M^{3}}\right) f}{B\left(f_{\max }\right)\left(\frac{f}{f_{\max }}\right)^{4.2}\left(1-\frac{f}{f_{\max }}\right)} .
$$

This fit to the numerical calculations typically agrees to within $10 \%$ with a maximum error of approximately $30 \%$ when $f$ is close to $f_{\text {max }}$.

Common to all the mechanisms which produce supernovae is a weak signal and a small number of cycles in the gravitational waves observed. Distinguishing these waveforms from non-Gaussian noise in the interferometers requires that signals from different interferometers be correlated. In the event that the signal is strong enough to be observed by both the 4 kilometer and 2 kilometer interferometers at Hanford, then the ratio of the gravitational strain produced in the two interferometers should be $2: 1$, increasing the confidence that the observation is that of a gravitational wave.

6.2. Compact binary sources. The inspiral and coalescence of compact binary systems composed of neutron stars and black holes are among the most promising sources of gravitational waves for LIGO to detect. The sensitivity of the initial LIGO interferometers will allow a coalescing pair of neutron stars to be detected out to 20 megaparsecs. A pair of 10 solar mass black holes could be detected at roughly 100 megaparsecs Detection of this type of source is greatly enhanced by the fact that the waveforms are known to a very high precision. This allows for the use of Wiener optimal filtering. The accuracy of the waveform templates of optimal filtering play a crucial role since a typical binary inspiral will advance by thousands of cycles as it sweeps upward in frequency through the 
LIGO bandwidth. Phase coherence must be maintained between the theoretical waveform and the signal detected by LIGO to no less than a quarter of a cycle in order for the optimal filtering technique to produce a strong match. To achieve this level of accuracy in the theoretical templates, it will be necessary to generate them using the second postNewtonian (2PN) waveforms [24]. The formulae for the $h_{\times}$and $h_{+}$waveforms to this order are given by

$$
\begin{gathered}
h_{+, \times}(t)=\frac{2 G m \eta}{c^{2} r}\left(\frac{G m \omega}{c^{3}}\right)^{\frac{2}{3}} \\
{\left[H_{+, \times}^{(0)}+\sqrt{x} H_{+, \times}^{\left(\frac{1}{2}\right)}+x H_{+, \times}^{(1)}+\sqrt[3]{x} H_{+, \times}^{\left(\frac{3}{2}\right)}+x^{2} H_{+, \times}^{(2)}\right]}
\end{gathered}
$$

where the brackets involve a post-Newtonian expansion whose various post-Newtonian terms are given for the plus polarization by

$$
\begin{aligned}
& H_{+}^{(0)}=-\left(1+c_{\imath}^{2}\right) \cos 2 \psi \\
& H_{+}^{\left(\frac{1}{2}\right)}=-\frac{s_{\imath}}{8} \frac{\delta m}{m}\left(\left(5+c_{\imath}^{2}\right) \cos \psi-9\left(1+c_{\imath}^{2}\right) \cos 3 \psi\right) \\
& H_{+}^{(1)}=\frac{1}{6}\left(\left(19+9 c_{\imath}^{2}-2 c_{\imath}^{4}\right)-\eta\left(19-11 c_{\imath}^{2}-6 c_{\imath}^{4}\right)\right) \cos 2 \psi \\
& -\frac{4}{3} s_{\imath}^{2}\left(1+c_{\imath}^{2}\right)(1-3 \eta) \cos 4 \psi \\
& H_{+}^{\left(\frac{3}{2}\right)}=\frac{s_{\imath}}{192} \frac{\delta m}{m}\left[\left(\left(57+60 c_{\imath}^{2}-c_{\imath}^{4}\right)-2 \eta\left(49-12 c_{\imath}^{2}-c_{\imath}^{4}\right)\right) \cos \psi\right. \\
& -\frac{27}{2}\left(\left(73+40 c_{\imath}^{2}-9 c_{\imath}^{4}\right)-2 \eta\left(25-8 c_{\imath}^{2}-9 c_{\imath}^{4}\right)\right) \cos 3 \psi \\
& \left.+\frac{625}{2}(1-2 \eta) s_{\imath}^{2}\left(1+c_{\imath}^{2}\right) \cos 5 \psi\right]-2 \pi\left(1+c_{\imath}^{2}\right) \cos 2 \psi \\
& H_{+}^{(2)}=\frac{1}{120}\left[\left(22+396 c_{\imath}^{2}+145 c_{\imath}^{4}-5 c_{\imath}^{6}\right)\right. \\
& +\frac{5}{3} \eta\left(706-216 c_{\imath}^{2}-251 c_{\imath}^{4}+15 c_{\imath}^{6}\right) \\
& \left.-5 \eta^{2}\left(98-108 c_{\imath}^{2}+7 c_{\imath}^{4}+5 c_{\imath}^{6}\right)\right] \cos 2 \psi \\
& +\frac{2}{15} s_{\imath}^{2}\left[\left(59+35 c_{\imath}^{2}-8 c_{\imath}^{4}\right)-\frac{5}{3} \eta\left(131+59 c_{\imath}^{2}-24 c_{\imath}^{4}\right)\right. \\
& \left.+5 \eta^{2}\left(21-3 c_{\imath}^{2}-8 c_{\imath}^{4}\right)\right] \cos 4 \psi-\frac{81}{40}\left(1-5 \eta+5 \eta^{2}\right) s_{\imath}^{4}\left(1+c_{\imath}^{2}\right) \cos 6 \psi \\
& +\frac{s_{\imath}}{40} \frac{\delta m}{m}\left[\left(11+7 c_{\imath}^{2}+10\left(5+c_{\imath}^{2}\right) \ln 2\right) \sin \psi-5 \pi\left(5+c_{\imath}^{2}\right) \cos \psi\right. \\
& \left.-27(7-10 \ln (3 / 2))\left(1+c_{\imath}^{2}\right) \sin 3 \psi+135 \pi\left(1+c_{\imath}^{2}\right) \cos 3 \psi\right]
\end{aligned}
$$

and for the cross polarization by

$$
\begin{aligned}
& H_{\times}^{(0)}=-2 c_{\imath} \sin 2 \psi \\
& H_{\times}^{\left(\frac{1}{2}\right)}=-\frac{3}{4} s_{\imath} c_{\imath} \frac{\delta m}{m}(\sin \psi-3 \sin 3 \psi) \\
& H_{\times}^{(1)}=\frac{c_{\imath}}{3}\left(\left(17-4 c_{\imath}^{2}\right)-\eta\left(13-12 c_{\imath}^{2}\right)\right) \sin 2 \psi-\frac{8}{3}(1-3 \eta) c_{\imath} s_{\imath}^{2} \sin 4 \psi
\end{aligned}
$$




$$
\begin{aligned}
H_{\times}^{\left(\frac{3}{2}\right)}= & \frac{s_{\imath} c_{\imath}}{96} \frac{\delta m}{m}\left[\left(\left(63-5 c_{\imath}^{2}\right)-2 \eta\left(23-5 c_{\imath}^{2}\right)\right) \sin \psi\right. \\
& -\frac{27}{2}\left(\left(67-15 c_{\imath}^{2}\right)-2 \eta\left(19-15 c_{\imath}^{2}\right)\right) \sin 3 \psi \\
& \left.+\frac{625}{2}(1-2 \eta) s_{\imath}^{2} \sin 5 \psi\right]-4 \pi c_{\imath} \sin 2 \psi \\
H_{\times}^{(2)}= & \frac{c_{\imath}}{60}\left[\left(68+226 c_{\imath}^{2}-15 c_{\imath}^{4}\right)+\frac{5}{3} \eta\left(572-490 c_{\imath}^{2}+45 c_{\imath}^{4}\right)\right. \\
& \left.-5 \eta^{2}\left(56-70 c_{\imath}^{2}+15 c_{\imath}^{4}\right)\right] \sin 2 \psi \\
& +\frac{4}{15} c_{\imath} s_{\imath}^{2}\left(\left(55-12 c_{\imath}^{2}\right)-\frac{5}{3} \eta\left(119-36 c_{\imath}^{2}\right)+5 \eta^{2}\left(17-12 c_{\imath}^{2}\right)\right) \sin 4 \psi \\
& -\frac{81}{20}\left(1-5 \eta+5 \eta^{2}\right) c_{\imath} s_{\imath}^{4} \sin 6 \psi \\
& -\frac{3}{20} s_{\imath} c_{\imath} \frac{\delta m}{m}[(3+10 \ln 2) \cos \psi+5 \pi \sin \psi \\
& -9(7-10 \ln (3 / 2)) \cos 3 \psi-45 \pi \sin 3 \psi] .
\end{aligned}
$$

The notation used is the following. The post-Newtonian expansion parameter is $x=$ $\left(G m \omega / c^{3}\right)^{2 / 3}$ where $\omega$ is the orbital frequency for the circular orbits, accurate to order $2 \mathrm{PN}$. The total mass is $m=m_{1}+m_{2}$ and $\eta=m_{1} m_{2} / m^{2}$. The constants $c_{\imath}=\cos \imath$ and $s_{\imath}=\sin \imath$ are defined using the angle of inclination $\imath$ of the orbital plane with respect to the line of sight. The phase variable $\psi$ is defined by

$$
\psi=\phi-\frac{2 G m \omega}{c^{3}} \ln \left(\frac{\omega}{\omega_{\circ}}\right) .
$$

The time dependence of the function $\phi$ and its time derivative $\omega$ are given by

$$
\begin{aligned}
\phi(t)= & \phi_{c}-\frac{1}{\eta}\left[\Theta^{\frac{5}{8}}(t)+\left(\frac{3715}{8064}+\frac{55}{96} \eta\right) \Theta^{\frac{3}{8}}(t)-\frac{3 \pi}{4} \Theta^{\frac{1}{4}}(t)\right. \\
& \left.+\left(\frac{9275495}{14450688}+\frac{284875}{258048} \eta+\frac{1855}{2048} \eta^{2}\right) \Theta^{\frac{1}{8}}(t)\right]
\end{aligned}
$$

where the constant $\phi_{c}$ is the phase at $t_{c}$, the time of coalescence,

$$
\begin{aligned}
\omega(t)= & \frac{c^{3}}{8 G m}\left[\Theta^{-\frac{3}{8}}(t)+\left(\frac{743}{2688}+\frac{11}{32} \eta\right) \Theta^{-\frac{5}{8}}(t)-\frac{3 \pi}{10} \Theta^{-\frac{3}{4}}(t)\right. \\
& \left.+\left(\frac{1855099}{14450688}+\frac{56975}{258048} \eta+\frac{371}{2048} \eta^{2}\right) \Theta^{-\frac{7}{8}}(t)\right]
\end{aligned}
$$

and where the dimensionless time variable $\Theta$ is given by

$$
\Theta(t)=\frac{c^{3} \eta}{5 G m}\left(t_{c}-t\right) .
$$

The particular choice for $\omega_{\circ}$ is physically irrelevant since it relates to the definition of the origin of time in the wave zone. A convenient choice is one which is the same for all templates and relates to the detector characteristics, such as the choice of $\omega_{\circ}=\pi f_{s c}$ where $f_{s c}$ corresponds to the seismic cutoff frequency for the interferometer.

The procedure for Wiener optimal filtering in the LIGO data analysis is to begin with the output $o(t)=h(t)+n(t)$ from the interferometer. For any second post-Newtonian 
template waveform, $h_{2 P N}(t)$, the optimal filter $\tilde{q}(f)$ is constructed by

$$
\tilde{q}(f)=\frac{\tilde{h}_{2 P N}(f)}{S_{n}(f)}, \quad \tilde{h}_{2 P N}(f)=\int_{-\infty}^{+\infty} h_{2 P N}(t) e^{i 2 \pi f t} d t
$$

where $S_{n}(f)$ is the noise spectral density defined as $\tilde{h}_{n o i s e}^{2}(f)$. The time domain representation of the Wiener optimal filter $q(t)$ is then determined by the Fourier transform

$$
q(t)=\int_{-\infty}^{+\infty} \tilde{q}(f) e^{-i 2 \pi f t} d t .
$$

This is then correlated to the output $o(t)$ from the interferometer

$$
\rho(t)=\int_{-\infty}^{+\infty} o(\tau) q(\tau+t) d \tau
$$

which through a useful property of Fourier transforms, can be rewritten as

$$
\rho(t)=\int_{-\infty}^{+\infty} \tilde{o}(f) \tilde{q}^{*}(f) e^{i 2 \pi f t} d f
$$

Equation 116 is the most efficient to implement on a computer. In practice the analysis with Wiener optimal filters will proceed as follows. The optimal filter will be constructed using Equation 113 for each second post-Newtonian waveform template by numerical means. The Fourier transform for the interferometer will also be calculated by numerical methods. Equation 116 will be evaluated using a fast Fourier transform (FFT), and the resulting $\rho(t)$ will be compared to at threshold.

The remaining issue for data analysis of the compact binary inspiral search is the determination of the number of waveform templates to use in the Wiener optimal filtering. This has been researched extensively [25, 26]. Findings indicate that for both the postNewtonian and the second post-Newtonian waveforms [27], the number of templates needed for the initial LIGO sensitivity is approximated by

$$
\mathcal{N} \simeq 2.4 \times 10^{5}\left(\frac{\mathcal{L}}{0.1}\right)^{-1}\left(\frac{M_{\min }}{0.2 \mathrm{M}_{\odot}}\right)^{-2.7}\left(\frac{\hat{f}}{200 H z}\right)^{-2.5}
$$

and for the projected advanced LIGO sensitivity, approximated by

$$
\mathcal{N} \simeq 7.6 \times 10^{6}\left(\frac{\mathcal{L}}{0.1}\right)^{-1}\left(\frac{M_{\min }}{0.2 \mathrm{M}_{\odot}}\right)^{-2.7}\left(\frac{\hat{f}}{70 H z}\right)^{-2.5}
$$

where $\mathcal{L}$ is the excepted fraction of signals lost in the analysis, $M_{\min }$ is the mass of the smaller of the binary companions, $\mathrm{M}_{\odot}$ is one solar mass, and $\hat{f}$ is the frequency at which the interferometer is most sensitive (roughly $140 \mathrm{~Hz}$ for the initial LIGO interferometers).

Based on the computational requirements of fast Fourier transforms [28], the computing power necessary to carry out the data analysis for the compact binary inspiral search has been estimated using a single pass, non-hierarchical scheme [26]. The CPU requirements for the initial LIGO sensitivity are

$$
\mathcal{P} \simeq 30 \times 10^{9} \operatorname{FLOPS}\left(\frac{\mathcal{L}}{0.1}\right)^{-1}\left(\frac{M_{\text {min }}}{0.2 \mathrm{M}_{\odot}}\right)^{-2.7}\left(\frac{\hat{f}}{200 H z}\right)^{-1.5}
$$


and for the projected advanced LIGO sensitivity, approximated by

$$
\mathcal{P} \simeq 400 \times 10^{9} \operatorname{FLOPS}\left(\frac{\mathcal{L}}{0.1}\right)^{-1}\left(\frac{M_{\text {min }}}{0.2 \mathrm{M}_{\odot}}\right)^{-2.7}\left(\frac{\hat{f}}{70 H z}\right)^{-1.5}
$$

For the initial LIGO sensitivity, each detector would require 40 GFLOPS of computing power to analyze the $5.9 \times 10^{5}$ waveform templates if a 10 percent loss of events was acceptable, and searches were carried out only for binary systems where the smaller companion had a mass of no less than $0.2 \mathrm{M}_{\odot}$. Computing power on this scale is available today and will become more easily accessible in the future, but this is a scale that becomes difficult to manage in an on-line data analysis system, especially in the early phases of observation. A balance between reduced on-line template searches and full off-line template search will probably be adopted by LIGO.

6.3. Periodic (pulsar) sources. Rotating neutron stars which are observationally known to exist as pulsars, will produce gravitational waves as a result of asymmetries about the axis of rotation [29]. These deviations from axisymmetry could arise from accretion of matter, solidification of the crust as it enters a final state during formation, as the result of "starquakes" or through complex interactions of magnetic fields. Gravitational waves will be produced at several frequencies. The star will radiate at twice its orbital frequency $\left(f_{\text {rot }}\right)$ as a result of the ellipticity induced by this asymmetry. If however, the principle axis of the star's moment of inertia tensor is misaligned with the rotation axis by a small angle $\theta$, a precession will exist, which causes the star to radiate at sideband frequencies to the rotational frequency. This will also introduce radiation at a frequency of $f_{\text {rot }}+f_{\text {prec }}$. For typical neutron star masses the characteristic amplitude of the gravitational waves are

$$
h_{c} \simeq 6 \times 10^{-25}\left(\frac{f_{\text {rot }}}{500 \mathrm{~Hz}}\right)^{2}\left(\frac{1 \mathrm{kpc}}{r}\right)\left(\frac{\epsilon_{e} \text { or } \theta \epsilon_{\text {prec }}}{10^{-6}}\right) .
$$

This is an extremely weak signal. In order to detect the signal it must be integrated for long periods of time. However, for any integration time having a chance of detecting the signal, there will be Doppler shift effects on the pulsar's gravitational wave frequencies.

There will be a gradual spin down of the radiating frequencies from both electromagnetic and gravitational wave emissions. This process is expected to lead to the glitches seen on a timescale of roughly once every four months, at which time the parameters driving the gravitational wave frequencies will change. For this reason it is very likely that integration times to enhance the signal to noise ratio will not be longer than mean times between glitches.

The fundamental approach to analysis is to take the Fourier transform of the interferometer output. Both of these effects cause the spectral power of the pulsar's gravitational wave signal to be spread out over adjacent bins in the frequency representation. To correct this, each location on the sky of a specific size, denoted as a "patch", must be corrected for the Earth's motion by a transformation to an inertial frame of reference. The effects of frequency spindown must also be taken into account by a similar transformation. The procedure for determining the number of patches (inclusive of the spindown) needed to accurately detect the pulsar has been determined by a method analogous to the metric 


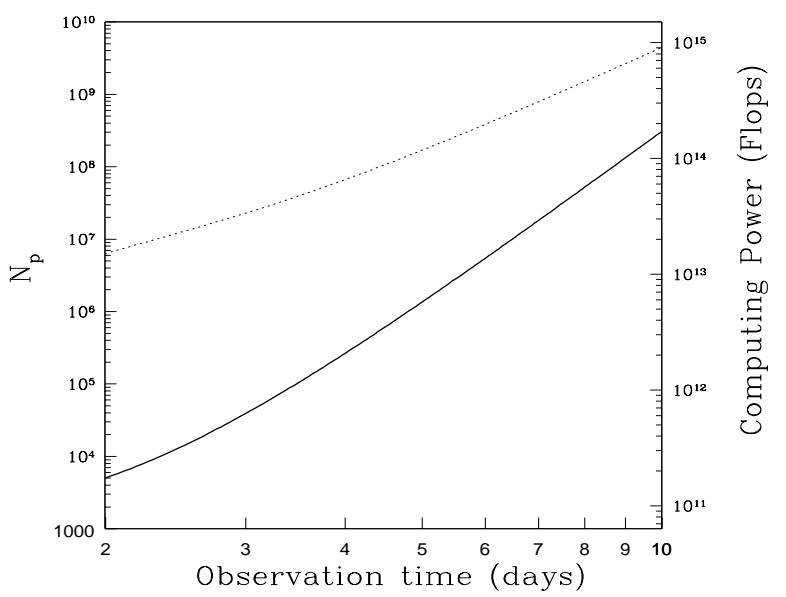

Figure 13: All sky pulsar search: Dotted line represents the number of patches needed to carry out search. The solid line shows for a given computing power, the optimized observation time with respect to loss of signals (not shown) which achieves the maximum signal to noise ratio.

method used to determine the number of binary inspiral templates [30]. The number is enormous for an "all sky search." For frequencies below $200 \mathrm{~Hz}$ and for observation times less than 10 days it is approximated by

$$
\mathcal{N} \simeq 4 \times 10^{5}\left(\frac{f_{\max }}{200 \mathrm{~Hz}}\right)^{2}\left(\frac{40 \text { years }}{\tau}\right)^{3}\left(\frac{0.3}{M_{\mathrm{o}}^{\max }}\right)^{2}\left(\frac{T_{\text {obs }}}{1 \text { day }}\right)^{5}
$$

where $f_{\max }$ is the maximum gravitational wave frequency searched, $\tau$ is the spindown rate for the pulsar, $M_{\circ}^{\max }$ is the maximum match between the signal and the patch and $T_{o b s}$ is the observation time in days.

Applying the computational characteristics of the fast Fourier transform to this problem, the computing power required to do the all sky search with a high assurance of detection is given by

$$
\mathcal{P} \simeq 2^{15} \mathcal{N}\left(\frac{f_{\max }}{1 \mathrm{kHz}}\right)\left(1+\frac{\ln \left(\frac{f_{\max }}{1 \mathrm{kHz}}\right)\left(\frac{T_{\text {obs }}}{10^{6} \mathrm{sec}}\right)}{31}\right)
$$

where $\mathcal{N}$ is the number of patches from Equation 122, and $T_{o b s}$ is the observation time in seconds. This is not the most efficient use of computing power. An optimization, which gives the best signal to noise, between the observational time required and the matching between the patches and the pulsar signal for a specified computing power is shown by the solid curve of Figure 13.

For signal integration times of any length, this becomes a many teraflop computing problem. While teraflop computers are on the horizon, they are not in the scope of on-line analysis plans for the initial LIGO detectors.

6.4. Stochastic background. Stochastic background searches will be used to place upper limits on the energy density of stochastic gravitational waves [31,32]. This background 
gravitational signal is analogous to the $3^{\circ}$ Kelvin microwave background in the electromagnetic spectrum. The precise definition of this energy density in terms of the critical energy density needed to close the universe $\rho_{\text {critical }}$ is

$$
\Omega_{G W}(f)=\frac{1}{\rho_{\text {critical }}} \frac{d \rho_{G W}}{d \ln f} .
$$

Sources of the stochastic background include the random superposition of gravitational waves from binary star systems, decaying cosmic strings, first-order phase transitions in the early Universe, and parametric amplification of quantum mechanical zero-point fluctuations in the metric tensor during inflation. The predicted strengths of all of these sources is highly uncertain, and given by the characteristic amplitude with Hubble constant $H_{\circ}$ by

$$
\tilde{h}_{c}(f)=1.3 \times 10^{-20}\left(\frac{H_{\circ}}{100 \mathrm{~km} / \mathrm{s} / \mathrm{Mpc}}\right) \sqrt{\Omega_{G W}}\left(\frac{100 \mathrm{~Hz}}{f}\right) .
$$

Placing upper limits on the energy density of the stochastic background or more importantly, the detection of the stochastic background, could introduce a wealth of information to better our understanding of the Universe. LIGO with its two detectors in Washington and Louisiana very nearly aligned provides for the optimal orientation of two detectors for stochastic background searches. The proposed VIRGO - GEO orientation minimizes that detector pair's sensitivity to stochastic background searches amplifying the role that LIGO will play in this type of signal search.

The stochastic background signal will be buried in the noise sources of a single detector and cannot be detected unless the signal strength is significantly larger than currently predicted. However, for two detectors with no correlated sources of noise, the only correlated fluctuations in the detector pair will be those due to stochastic gravitational waves. Therefore the detection scenario for the stochastic background involves a properly weighted correlation of the strain outputs from two detectors having uncorrelated noise sources. The proper weighting is given by the optimal filter

$$
\left(\frac{S}{N}\right)^{2}=\frac{9 H_{\circ}^{4}}{50 \pi^{4}} \tau \int_{0}^{\infty} \frac{\gamma^{2}(f) \Omega_{G W}^{2}(f)}{f^{6} S_{n 1}(f) S_{n 2}(f)} d f
$$

where $S_{n 1}(f)$ and $S_{n 2}(f)$ are the noise power spectral densities of each of the detectors, $\tau$ is the integration time, $H_{\circ}$ is the Hubble constant in units of $\sec ^{-1}$ and $\gamma(f)$ is the overlap reduction function given by

$$
\gamma(f)=\frac{5}{8 \pi} \oint_{S^{2}} e^{i 2 \pi f \hat{\Omega} \cdot \Delta \vec{x} / c}\left(F_{1}^{+} F_{2}^{+}+F_{1}^{\times} F_{2}^{\times}\right) d \hat{\Omega}
$$

and is a function of the separation $\Delta \vec{x}$ between the two detectors and the response $F_{i}^{+, \times}$ of the detectors to the gravitational wave polarizations.

$$
F_{i}^{+, \times}=\frac{1}{2}\left(\hat{X}_{i}^{a} \hat{X}_{i}^{b}-\hat{Y}_{i}^{a} \hat{Y}_{i}^{b}\right) e_{a b}^{+, \times}(\hat{\Omega})
$$

where $\hat{\Omega}$ is the unit vector on the two-sphere, $X_{i}^{a}$ and $Y_{i}^{a}$ are the directions of the detector $i$ arms and $e_{a b}^{+, \times}(\hat{\Omega})$ are the polarization tensors.

This dependency is basically due to the fact that the arms will on average move strongly in phase only for gravitational waves having wavelengths longer than twice the 
distance between the detector pair. For the Hanford - Livingston separation of 3030 kilometers, this corresponds to stochastic signals of frequency less than approximately 50 Hz. Even though the optimal filter for stochastic signal detection peaks strongly below this frequency, the full spectral range of the interferometers is expected to be used in the search and non-trivial weighting carried out to at least $1 \mathrm{kHz}$.

In order to properly carry out the two detector signal correlation using the optimal filter, the pre-whitened signals from the two detectors will need to be unwhitened to bring the two signals back into the proper phase relationships for the gravitational wave, unless the pre-whitening filters from the two detectors are identical in their phase response over the frequencies of interest. This will require that the full amplitude and phase characteristics of the pre-whitening filters be recorded and available to the stochastic data analysis. Additionally, since the optimal filter is peaked for frequencies below $50 \mathrm{~Hz}$, it is important that the pre-whitening filters do not zero out the strain signal at these lower frequencies. In fact, the dependency on the frequency in the signal to noise ratio is proportional to $f^{-3}$. The signal to noise ratio also increases with time. Searches will most likely utilize months if not years of data. However, this type of search does not require the vast computer power of the periodic search since the strain data does not require doppler shifts and data can be handled in smaller sections.

Intrasite searches for stochastic signals such as with the two interferometers at Hanford are not expected to be straight forward to implement due to the strong correlations in the common noise sources such as power lines, gas column density fluctuations in the beam tube, and seismic noise. Even if these correlated noises were recorded, there would be no way to distinguish them from the stochastic background signals. However, since the two detectors are co-located, the search would encompass lower frequency stochastic gravitational waves where the optimal filter is more strongly peaked for the initial LIGO sensitivity.

6.5. Advanced analysis methods. The methods described in the searches for expected sources of gravitational waves are considered the "baseline" techniques to be used by LIGO. Other methods for data analysis are being considered and hold much promise for faster on-line analysis and the search for gravitational waves from sources with unknown or poorly known waveforms. Among these are the Gabor and wavelet transforms [33] in which the analysis is carried out in a "time + frequency" representation. Other methods include adaptive line enhancement where the filter is self adjusting based on the input data sequence [34]. In all of these methods, questions of robustness have caused them to remain in the background, while the "brute-force" methods are being planned for use in the initial LIGO data analysis. Still, they hold great promise and deserve consideration.

7. Acknowledgments. I would like to acknowledge my colleagues on the LIGO project for their effort in bringing together the work reviewed herein. I would also like to acknowledge Andrzej Królak of the Polish Academy of Sciences for his encouragement during the writing of this review. LIGO is supported by the National Science Foundation under the cooperative agreement PHY-9210038. 


\section{References}

[1] A. Abramovici, W. Althouse, R. Drever, Y. Gursel, S. Kawamura, F. Raab, D. Shoemaker, L. Seivers, R. Spero, K. Thorne, R. Vogt, R. Weiss, S. Whitcomb, M. Zuker, Science, 256, 325, (1992).

[2] K. S. Thorne, In S. E. Hawking and W. Isreal, editors, Three Hundred Years of Gravitation, 330, Cambridge University Press, (1987).

[3] R. A. Hulse and J. H. Taylor, Astrophys. J., 324, (1975).

[4] R. Epstein and R. V. Wagoner, Astrophys. J. 197, 717, (1975)

[5] R. V. Wagner and C. M. Will, Astrophys. J. 210, 764, (1976)

[6] T. A. Apostolatos, Phys. Rev. D 52, 605 (1995).

[7] Clifford M. Will and Alan G. Wiseman, to appear in Phys. Rev. D

[8] J. Giame, "Studies of Laser interferometer Design and a Vibration isolation System for Interferometric Gravitational Wave Detectors", Ph. D. thesis, MIT, (1995).

[9] C. N. Man, D. Shoemaker, M. Pham Tu and D. Dewey, Phys. Lett. A, 148, 8, (1990).

[10] Martin W. Regehr, "Signal Extraction and Control for an Interferemetric Gravitational Wave Detector", Ph. D thesis, Caltech, (1995).

[11] J. Giame, P. Saha, D. Shoemaker, L. Sievers, Rev. Sci. Instrum. 67 (1), 208, (1996).

[12] P. R. Saulson, Fundamentals of Interferometric Gravitational Wave Detectors, New Jersey: Wold Scientific, (1994).

[13] A. Gillespie and F. Raab, Phys. Lett. A, 178, 357, (1993).

[14] A. Gillespie and F. Raab, Phys. Rev. D, 52, 577, (1995).

[15] J. R. Hutchinson, J. Appl. Mech., 47, 901, (1980).

[16] V. B. Braginsky, V. P. Mitrofanov and O.A. Okhrimenko, JETP Lett., 55, 432, (1992).

[17] Malcolm B. Gray, Andrew J. Stevenson, Hans-A. Bachor and David E. McClelland, Optics Lett.,18, (10), 759, (1993).

[18] T. Lyons and M. Regehr, "Shot Noise in a Recycled Unbalanced LIGO", LIGO Technical Report, (1994).

[19] Michael E. Zucker and Stanley E. Whitcomb, "Measurement of Optical Path Fluctuations due to Residual Gas in the LIGO 40 Meter Interferometer", Marcel Grossman Proceedings, (1996).

[20] Scott A. Hughes and Kip S. Thorne, "Seismic Gravity Gradient Noise in Interferometric Gravitational Wave Detectors", in preparation, (1996).

[21] A. Burrows, J. Hayes and B. A. Fryxell, to appear in Astrophys. J.

[22] D. Lai and S. L. Shapiro, Astrophys. J., 442, 259, (1995).

[23] Dong Lai, private communication, (1996).

[24] Luc Blanchet, Bala R. Iyer, Clifford M. Will and Alan G. Wiseman, to appear in Class. and Quantum Grav.

[25] B. S. Sathyaprakash, Phys. Rev. D, 50, 7111, (1994).

[26] Benjamin J. Owen, Phys. Rev. D 536749 (1996).

[27] B. S. Sathyaprakash, private communication, (1996).

[28] B. F. Schutz, in The Detection of Gravitational Radiation, ed. D. Blair (Cambridge University Press, Cambridge, England, (1989).

[29] Kip Thorne, submitted to Proceedings of Snowmass 94 Summer Study on Particle and Nuclear Astrophysics and Cosmology, eds. E. W. Kolb and R. Peccei, World Scientific, Singapore, (1994). 
[30] Patrick R. Brady and Teviet Creighton, to be submitted to Phys. Rev. D, (1996)

[31] Eanna E. Flanagan, Phys. Rev. D, 48, 2389, (1993).

[32] B. Allen, to appear in Proceedings of the Les Houches School on Astrophysical Sources of Gravitational Radiation, ed. Jean-Alain Marck, Springer Verlag, (1996).

[33] Jean-Micheal Innocent and Bruno Torresani, this volume.

[34] David Nicholson, preprint, (1995). 